\title{
Fractional Partial Differential Equation: Fractional Total Variation and Fractional Steepest Descent Approach-Based Multiscale Denoising Model for Texture Image
}

\author{
Yi-Fei Pu, ${ }^{1,2}$ Ji-Liu Zhou, ${ }^{1}$ Patrick Siarry, ${ }^{3}$ Ni Zhang, ${ }^{4}$ and Yi-Guang Liu ${ }^{1}$ \\ ${ }^{1}$ School of Computer Science and Technology, Sichuan University, Chengdu 610065, China \\ ${ }^{2}$ State Key Laboratory of Networking and Switching Technology, Beijing University of Posts and Telecommunications, \\ Beijing 100876, China \\ ${ }^{3}$ Université de Paris 12 (LiSSi, E.A. 3956), 61 avenue du Général de Gaulle, 94010 Créteil Cedex, France \\ ${ }^{4}$ Library of Sichuan University, Chengdu 610065, China
}

Correspondence should be addressed to Yi-Fei Pu; puyifei_007@163.com

Received 14 June 2013; Accepted 11 August 2013

Academic Editor: Juan J. Trujillo

Copyright (c) 2013 Yi-Fei Pu et al. This is an open access article distributed under the Creative Commons Attribution License, which permits unrestricted use, distribution, and reproduction in any medium, provided the original work is properly cited.

The traditional integer-order partial differential equation-based image denoising approaches often blur the edge and complex texture detail; thus, their denoising effects for texture image are not very good. To solve the problem, a fractional partial differential equation-based denoising model for texture image is proposed, which applies a novel mathematical method-fractional calculus to image processing from the view of system evolution. We know from previous studies that fractional-order calculus has some unique properties comparing to integer-order differential calculus that it can nonlinearly enhance complex texture detail during the digital image processing. The goal of the proposed model is to overcome the problems mentioned above by using the properties of fractional differential calculus. It extended traditional integer-order equation to a fractional order and proposed the fractional Green's formula and the fractional Euler-Lagrange formula for two-dimensional image processing, and then a fractional partial differential equation based denoising model was proposed. The experimental results prove that the abilities of the proposed denoising model to preserve the high-frequency edge and complex texture information are obviously superior to those of traditional integral based algorithms, especially for texture detail rich images.

\section{Introduction}

Fractional calculus has been an important branch of mathematical analysis over the last 300 years [1-4]; however, it is still little known by many mathematicians and physical scientists in both the domestic and overseas engineering fields. Fractional calculus of the Hausdorff measure is not well established after more than 90 years studies $[5,6]$, whereas fractional calculus in the Euclidean measure seems more completed. So, Euclidean measure is commonly required in mathematics $[5,6]$. In general, fractional calculus in the Euclidean measure extends the integer step to a fractional step. Random variable of physical process in the Euclidean measure can be deemed to be the displacement of particles by random movement; thus, fractional calculus can be used for the analysis and processing of the physical states and processes in principle [7-15]. Fractional calculus has one obvious feature, that is, that most fractional calculus is based on a power function and the rest is based on the addition or production of a certain function and a power function [1-6]. It is possible that this feature indicates some changing law of nature. Scientific research has proved that the fractionalorder or dimensional mathematical approach provides the best description for many natural phenomena [16-19]. Fractional calculus in the Euclidean measure has been used in many fields, including diffusion process, viscoelasticity theory, and random fractal dynamics. Methods to apply fractional calculus to modern signal analysis and processing [18-30], especially to digital image processing [31-38], are an emerging branch to study, which has been seldom explored. 
Integer-order partial differential equation-based image processing is an important branch in the field of image processing. By exploring the essence of image and image processing, people tend to reconstruct the traditional image processing approaches through strictly mathematical theories, and it will be a great challenge to practical-oriented traditional image processing. Image denoising is a significant research branch of integer-order partial differential equation-based image processing, with two kinds of denoising approach: the nonlinear diffusion-based method and the minimum energy norm-based variational method [39-42]. They have two corresponding models, which are the anisotropic diffusion proposed by Perona and Malik [43] (Perona-Malik or PM) and the total variation model proposed by Rudin et al. [44] (Rudin-Osher-Fatemi or ROF). The PM model simulates the denoising process by a thermal energy diffusion process and the denoising result is the balanced state of thermal diffusion, while the ROF model describes the same thermal energy by a total variation. In further study, some researchers have applied the PM model and the ROF model to color images [45, 46], discussed the selection of the parameters for the models [47-51], and found the optimal stopping point in iteration process $[52,53]$. Rudin and his team proposed a variable time step method to solve the Euler-Lagrange equation [44]. Vogel and Oman proposed improving the stability of ROF model by a fixed point iteration approach [54]. Darbon and Sigelle decomposed the original problems into independent optimal Markov random fields by using level set methods and obtained globally optimal solution by reconstruction [55-57]. Wohlberg and Rodriguez proposed to solve the total variation by using an iterative weighted norm to improve the computing efficiency [58]. Meanwhile, Catté et al. proposed to perform a Gaussian smoothing process in the initial stage to improve the suitability of the PM model [59]. However, PM model and ROF model have some obvious defects in image denoising; that is, they can easily lose the contrast and texture details and can produce staircase effects $[39,60,61]$. Some improved models have been proposed to solve these problems. To maintain the contrast and texture details, some scholars have proposed to replace the $L^{2}$ norm with the $L^{1}$ norm [62-65], while Osher et al. proposed an iterative regularization method [66]. Gilboa et al. proposed a denoising method using a numerical adaptive fidelity term that can change with the space [67]. Esedoglu and Osher proposed to decompose images using the anisotropic ROF model and retaining certain edge directional information [68]. To remove the staircase effects, Blomgren et al. proposed to extend the total variation denoising model by changing it with gradients $[69,70]$. Some scholars introduced high-order derivative to energy norm [71-76]. Lysaker et al. integrated high-order deductive to original ROF model $[77,78]$, while other scholars proposed a two-stage denoising algorithm, which smoothes the corresponding vector field first and then fits it by using the curve surface [79, 80]. The above methods have provided some improvements in maintaining contrast and texture details and removing the staircase effect, but they still have some drawbacks. First, the improved algorithms have greatly increased calculation complexity for real-time processing and excessive storage and computational requirements will lead them to be impractical. Second, the above algorithms are essentially integer-order differential based algorithm, and thus they may cause the edge field to be somewhat fuzzy and the texture-preservation effect to be less effective than expected.

We therefore propose to introduce a new mathematical method-fractional calculus to the denoising field for texture image and implementing a fractional partial differential equation to solve the above problems by the integer-order partial differential equation-based denoising algorithms [23, 33-38]. Guidotti and Lambers [81] and Bai and Feng [82] have pushed the classic anisotropic diffusion model to the fractional field, extended gradient operator of the energy norm from first-order to fractional-order, and numerically implemented the fractional developmental equation in the frequency domain, which has some effects on image denoising. However, the algorithm still has certain drawbacks. First, it simply took the gradient operator of the energy norm from first order to fractional order and still cannot essentially solve the problem of how to nonlinearly maintain the texture details via the anisotropic diffusion. Therefore, the texture information is not retained well after denoising. Second, the algorithm does not include the effects of the fractional power of the energy norm and the fractional extreme value on nonlinearly maintaining texture details. Third, the method does not deduce the corresponding fractional Euler-Lagrange formula according to fractional calculus features and directly replace it according to the complex conjugate transpose features of the Hilbert adjoint operator. It greatly increased the complex of the numerical implementation of the fractional developmental equation in frequency field. Finally, the transition function of fractional calculus in Fourier transform domain is $(i \omega)^{v}$. Its form looks simple, but the Fourier's inverse transform of $(i \omega)^{v}$ belongs to the first kind of Euler integral, which is difficult to calculate theoretically. The algorithm simply converted the first-order difference into the fractional-order difference in the frequency domain form and replaced the fractional differential operator, which has not solved the computing problem of the Euler integral of the first kind.

The properties of fractional differential are as follows [23, $24,38]$. First, the fractional differential of a constant is nonzero, whereas it must be zero under integer-order differential. Fractional calculus varies from a maximum at a singular leaping point to zero in the smooth areas where the signal is unchanged or not changed greatly; note that, by default, any integer-order differential in a smooth area is approximated to zero, which is the remarkable difference between the fractional differential and integer-order differential. Second, the fractional differential at the starting point of a gradient of a signal phase or slope is nonzero, which nonlinearly enhances the singularity of high-frequency components. With the increasing fractional order, the strengthening of the singularity of high-frequency components is also greater. For example, when $0<v<1$, the strengthening is less than when $v=1$. The integral differential is a special case of the fractional calculus. Finally, the fractional calculus along the slope is neither zero nor constant but is a nonlinear curve, while integer-order differential along slope is the constant. 
From this discussion, we can see that fractional calculus can nonlinearly enhance the complex texture details during the digital image processing. Fractional calculus can nonlinearly maintain the low-frequency contour features in smooth area to the furthest degree, nonlinearly enhance the highfrequency edge and texture details in those areas where gray level changes frequently, and nonlinearly enhance highfrequency texture details in those areas where gray level does not change obviously [23, 33-38].

A fractional partial differential equation-based denoising algorithm is proposed. The experimental results prove that it can not only preserve the low-frequency contour feature in the smooth area but also nonlinearly maintain the highfrequency edge and texture details both in the areas where gray level did not change obviously or change frequently. As for texture-rich images, the abilities for preserving the highfrequency edge and complex texture details of the proposed fractional based denoising model are obviously superior to the traditional integral based algorithms. The outline of the paper is as follows. First, it introduces three common-used definitions of fractional calculus, that is, Grümwald-Letnikov, Riemann-Liouville, and Caputo, which are the premise of the fractional developmental equation-based model. Second, we obtain fractional Green's formula for two-dimensional image by extending the classical integer-order to a fractional-order and also fractional Euler-Lagrange formula. On the basis, a fractional partial differential equation is proposed. Finally, we show the denoising capabilities of the proposed model by comparing with Gaussian denoising, fourth-order TV denoising, bilateral filtering denoising, contourlet denoising, wavelet denoising, nonlocal means noise filtering (NLMF) denoising, and fractional-order anisotropic diffusion denoising.

\section{Related Work}

The common-used definitions of fractional calculus in the Euclidean measure are Grümwald-Letnikov definition, Riemann-Liouville definition, and Caputo definition [1-6].

Grümwald-Letnikov defined that $v$-order differential of signal $s(x)$ can be expressed by

$$
\begin{aligned}
D_{G-L}^{v} s(x)= & \left.\frac{d^{v}}{[d(x-a)]^{v}} s(x)\right|_{G-L} \\
= & \lim _{N \rightarrow \infty}\left\{\frac{((x-a) / N)^{-v}}{\Gamma(-v)}\right. \\
& \left.\quad \times \sum_{k=0}^{N-1} \frac{\Gamma(k-v)}{\Gamma(k+1)} s\left(x-k\left(\frac{x-a}{N}\right)\right)\right\},
\end{aligned}
$$

where the duration of $s(x)$ is $[a, x]$ and $v$ is any real number (fraction included). $D_{G-L}^{v}$ denotes Grümwald-Letnikov defined fractional-order differential operator, and $\Gamma$ is Gamma function. Equation (1) shows that GrümwaldLetnikov definition in the Euclidean measure extends the step from integer to fractional, and thus it extends the order from integer differential to fractional differential. GrümwaldLetnikov defined fractional calculus is easily calculated, which only relates to the discrete sampling value of $s(x-k)(x-$ a) $/ N)$ ) that correlates to $s(x)$ and irrelates to the derivative or the integral value.

Riemann-Liouville defined the $v$-order integral when $v<$ 0 is shown as

$$
\begin{aligned}
D_{R-L}^{v} s(x) & =\left.\frac{d^{v}}{[d(x-a)]^{v}} s(x)\right|_{R-L} \\
& =\frac{1}{\Gamma(-v)} \int_{a}^{x}(x-\eta)^{-v-1} s(\eta) d \eta \\
& =\frac{-1}{\Gamma(-v)} \int_{a}^{x} s(\eta) d(x-\eta)^{-v}, \quad v \prec 0,
\end{aligned}
$$

where $D_{R-L}^{v}$ represents the Riemann-Liouville defined fractional differential operator. As for $v$-order differential when $v \geq 0, n$ satisfies $n-1<v \leq n$. Riemann-Liouville defined $v$-order differential can be given by

$$
\begin{aligned}
D_{R-L}^{v} s(x)= & \left.\frac{d^{v}}{[d(x-a)]^{v}} s(x)\right|_{R-L} \\
= & \left.\frac{d^{n}}{d x^{n}} \frac{d^{v-n}}{\left[d(x-a]^{v-n}\right.} s(x)\right|_{R-L} \\
= & \sum_{k=0}^{n-1} \frac{(x-a)^{k-v} s^{(k)}(a)}{\Gamma(k-v+1)} \\
& +\frac{1}{\Gamma(n-v)} \int_{a}^{x} \frac{s^{(n)}(\eta)}{(x-\eta)^{v-n+1}} d \eta, \quad 0 \leq v \prec n .
\end{aligned}
$$

Fourier transform of the $s(x)$ is expressed as

$$
\mathrm{FT}\left[D^{v} s(x)\right]=(i \omega)^{v} \mathrm{FT}[s(x)]-\sum_{k=0}^{n-1}(i \omega)^{k} \frac{d^{\nu-1-k}}{d x^{\nu-1-k}} s(0),
$$

where $i$ denotes imaginary unit and $\omega$ represents digital frequency. If $s(x)$ is causal signal, (4) can be simplified to read

$$
\mathrm{FT}\left[D^{v} s(x)\right]=(i \omega)^{v} \mathrm{FT}[s(x)]
$$

\section{Theoretical Analysis for Fractional Partial Differential Equation: Fractional Total Variation and Fractional Steepest Descent Approach Based Multiscale Denoising Model for Texture Image}

3.1. The Fractional Green Formula for Two-Dimensional Image. The premise of implementing Euler-Lagrange formula is to obtain the proper Green's formula [83]. We therefore extend the order of Green's formula from the integer to a fractional first in order to implement fractional EulerLagrange formula of two-dimensional image.

Consider $\Omega$ to be simply connected plane region, taking the piecewise smooth curve $C$ as a boundary; then the differintegrable functions $P(x, y)$ and $Q(x, y)[1-6]$ are continuous 


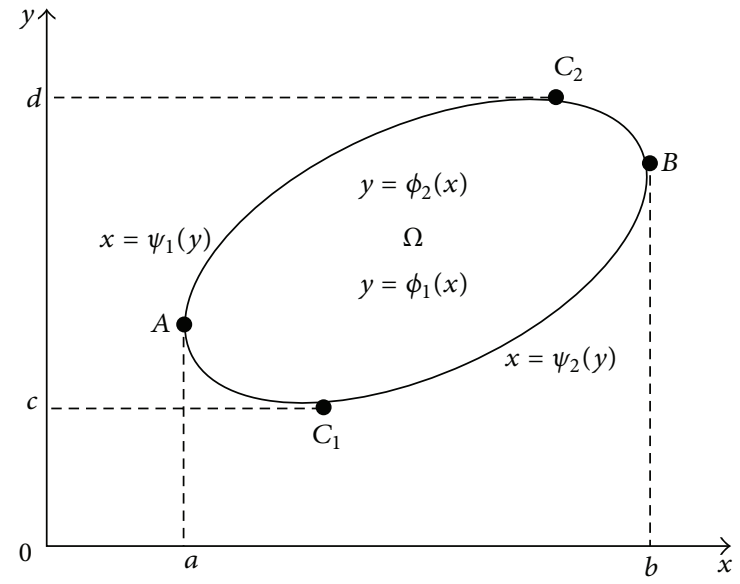

FIGURE 1: Simply connected space $\Omega$ and its smooth boundary curve C.

in $\Omega$ and $C$, and the fractional continuous partial derivatives for $x$ and $y$ exist. If we consider $D^{1}$ to represent the firstorder differential operator, then $D^{v}$ represents the $v$-order fractional differential operator, $I^{1}=D^{-1}$ denotes the firstorder integral operator, and $I^{v}=D^{-v}$ represents $v$-order fractional integral operator when $v>0$. Note that $\left(I_{x}^{v} I_{y}^{v}\right)_{\Omega}$ represents the $v$-order integral operator of curve surface in the $\Omega$ plane. $I_{C\left(A C_{1} B\right)}^{v}$ is the $v$-order integral operator in the $A C_{1} B$ section of curve $C$ along the direction of $\overrightarrow{A C_{1} B} . I_{C-}^{v}$ is $v$-order fractional integral operator in the closed curve $C$ along counter-clockwise direction. Consider that boundary $C$ is circled by two curves $y=\phi_{1}(x), y=\phi_{2}(x), a \leq x \leq b$ or $x=\psi_{1}(y), x=\psi_{2}(y), c \leq y \leq d$, as shown in Figure 1 .

As for differintegrable function $P(x, y)$ [1-6], when $P$ $D^{-v_{1}} D^{v_{1}} P \neq 0$, it follows that $D^{v_{1}} D^{v_{2}} P=D^{v_{1}+v_{2}} P-$ $D^{v_{1}+v_{2}}\left(P-D^{-v_{1}} D^{v_{1}} P\right)$. Thus, when $I_{x}^{v_{2}} I_{y}^{v_{2}} D_{y}^{v_{1}} P(x, y)=$ $I_{x}^{v_{2}}\left\{D_{y}^{v_{1}-v_{2}} P(x, y)-D_{y}^{v_{1}-v_{2}}\left[P(x, y)-D_{y}^{-v_{1}} D_{y}^{v_{1}} P(x, y)\right]\right\}$, it has

$$
\begin{aligned}
& \left(I_{x}^{v_{2}} I_{y}^{v_{2}}\right)_{\Omega} D_{y}^{v_{1}} P(x, y) \\
& ={ }_{a}^{b} I_{x}^{v_{2}} \phi_{\phi_{1}(x)}(x) I_{y}^{v_{2}} D_{y}^{v_{1}} P(x, y) \\
& ={ }_{a}^{b} I_{x}^{v_{2}}\left\{D_{y}^{v_{1}-v_{2}} P(x, y)\right. \\
& \left.\quad-D_{y}^{v_{1}-v_{2}}\left[P(x, y)-D_{y}^{-v_{1}} D_{y}^{v_{1}} P(x, y)\right]\right\}\left.\right|_{\phi_{1}(x)} ^{\phi_{2}(x)} \\
& =-I_{x}^{v_{2}}\left\{D_{y}^{v_{1}-v_{2}} P(x, y)\right. \\
& C\left(B C_{2} A\right) \\
& \left.\quad-D_{y}^{v_{1}-v_{2}}\left[P(x, y)-D_{y}^{-v_{1}} D_{y}^{v_{1}} P(x, y)\right]\right\} \\
& \quad-\quad I_{x}^{v_{2}}\left\{D_{y}^{v_{1}-v_{2}} P(x, y)\right. \\
& \left.\quad-D_{y}^{v_{1}-v_{2}}\left[P(x, y)-D_{y}^{-v_{1}} D_{y}^{v_{1}} P(x, y)\right]\right\} \\
& =-I_{x}^{v_{2}}\left\{D_{y}^{v_{1}-v_{2}} P(x, y)\right. \\
& \left.C-D_{y}^{v_{1}-v_{2}}\left[P(x, y)-D_{y}^{-v_{1}} D_{y}^{v_{1}} P(x, y)\right]\right\} .
\end{aligned}
$$

Similarly, it has

$$
\begin{aligned}
& \left(I_{x}^{v_{2}} I_{y}^{v_{2}}\right)_{\Omega} D_{x}^{v_{1}} Q(x, y) \\
& =\underset{y}{I_{y}^{v_{2}}}\left\{D_{x}^{v_{1}-v_{2}} Q(x, y)\right. \\
& \left.\quad \quad \quad-D_{x}^{v_{1}-v_{2}}\left[Q(x, y)-D_{x}^{-v_{1}} D_{x}^{v_{1}} Q(x, y)\right]\right\} .
\end{aligned}
$$

Fractional Green's formula of two-dimensional image can be expressed by

$$
\begin{aligned}
&\left(I_{x}^{v_{2}} I_{y}^{v_{2}}\right)_{\Omega}\left(D_{x}^{v_{1}} Q(x, y)-D_{y}^{v_{1}} P(x, y)\right) \\
&=I_{x}^{I_{2}}\left\{D_{y}^{v_{1}-v_{2}} P(x, y)\right. \\
&\left.\quad-D_{y}^{v_{1}-v_{2}}\left[P(x, y)-D_{y}^{-v_{1}} D_{y}^{v_{1}} P(x, y)\right]\right\} \\
&+I_{C_{-}}^{v_{2}}\left\{D_{x}^{v_{1}-v_{2}} Q(x, y)\right. \\
&\left.\quad \quad-D_{x}^{v_{1}-v_{2}}\left[Q(x, y)-D_{x}^{-v_{1}} D_{x}^{v_{1}} Q(x, y)\right]\right\} .
\end{aligned}
$$

When $D^{v_{1}}$ and $D^{-v_{1}}$ are reciprocal, that is, $\varphi-D^{-v_{1}} D^{v_{1}} \varphi=0$, it follows that $D^{v_{1}} D^{v_{2}} \varphi=D^{v_{1}+v_{2}} \varphi$. Then, (8) can be simplified to read

$$
\begin{aligned}
\left(I_{x}^{v_{2}} I_{y}^{v_{2}}\right)_{\Omega} & \left(D_{x}^{v_{1}} Q(x, y)-D_{y}^{v_{1}} P(x, y)\right) \\
& =I_{C-}^{I_{2}} D_{y}^{v_{1}-v_{2}} P(x, y)+{ }_{C-}^{I_{2}^{v_{2}}} D_{x}^{v_{1}-v_{2}} Q(x, y) .
\end{aligned}
$$

We know from (9) the following. First, when $v_{1}=v_{2}=v$, (9) can be simplied as $\left(I_{x}^{v} I_{y}^{v}\right)_{\Omega}\left(D_{x}^{v} Q(x, y)-D_{y}^{v} P(x, y)\right)=$ $\left(I_{x}^{v}\right)_{C_{-}} P(x, y)+\left(I_{y}^{v}\right)_{C_{-}} Q(x, y)$, which is the expression of fractional Green's formula in reference [84]. Second, when $v_{1}=v_{2}=1$, it follows that $\left(I_{x}^{1} I_{y}^{1}\right)_{\Omega}\left(D_{x}^{1} Q(x, y)-\right.$ $\left.D_{y}^{1} P(x, y)\right)=\left(I_{x}^{1}\right)_{C_{-}} P(x, y)+\left(I_{y}^{1}\right)_{C_{-}} Q(x, y)$. The classical integer-order Green's formula is the special case of fractional Green formula.

3.2. The Fractional Euler-Lagrange Formula for Two-Dimensional Image. To implement a fractional partial differential equation-based denoising model, we must obtain the fractional Euler-Lagrange formula first, and thus we furtherly deduce the fractional Euler-Lagrange formula for twodimensional image based on the above fractional Green's formula.

Consider the differintegrable numerical function in twodimensional space to be $u(x, y)$ and the differintegrable vector function to be $\vec{\varphi}(x, y)=i \varphi_{x}+j \varphi_{y}[1-6]$; the $v$-order fractional differential operator is $D^{v}=i\left(\partial^{v} / \partial x^{v}\right)+j\left(\partial^{v} /\right.$ $\left.\partial y^{v}\right)=i D_{x}^{v}+j D_{y}^{v}=\left(D_{x}^{v}, D_{y}^{v}\right)$. Here, $D^{v}$ is a type of linear operator. When $v=0$, then $D^{0}$ represents an equality operator, which is neither differential nor integral, where $i$ and $j$, respectively, represent the unit vectors in the $x$ - and $y$ directions. In general, the two-dimensional image region $\Omega$ is a rectangular simply connected space, and thus the piecewise 


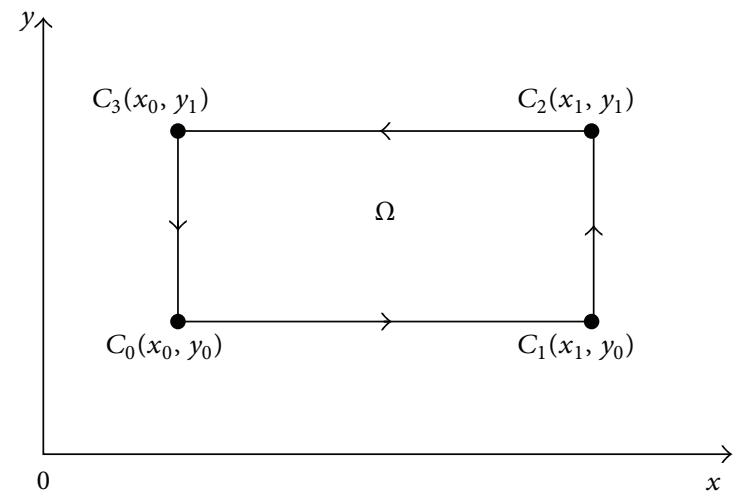

FIGURE 2: The two-dimensional simply connected space $\Omega$ and its piecewise smooth boundary curve $C$.

smooth boundary $C$ is also a rectangular closed curve, as shown in Figure 2.

Referring to (2), it follows that $I_{x}^{v} s(x, y)=(1 /$ $\Gamma(v)) \int_{a_{x}}^{x}(x-\eta)^{v-1} s(\eta, y) d \eta, I_{y}^{v} s(x, y)=(1 / \Gamma(v)) \int_{a_{y}}^{y}(y-$ $\zeta)^{v-1} s(x, \zeta) d \zeta$ and $I_{x}^{v} I_{y}^{v} s(x, y)=\left(1 / \Gamma^{2}(v)\right) \int_{a_{x}}^{x} \int_{a_{y}}^{y}(x-$ $\eta)^{v-1}(y-\zeta)^{\nu-1} s(\eta, \zeta) d \eta d \zeta$. From the fractional Green formula (8) and Figure 2, we can derive

$$
\begin{aligned}
\left(I_{x}^{v_{2}} I_{y}^{v_{2}}\right)_{\Omega} & \left(D_{x}^{v_{1}} Q(x, y)-D_{y}^{v_{1}} P(x, y)\right) \\
=I_{x}^{v_{2}}\left\{D_{y}^{v_{1}-v_{2}} P(x, y)\right. & \\
& \left.\quad-D_{y}^{v_{1}-v_{2}}\left[P(x, y)-D_{y}^{-v_{1}} D_{y}^{v_{1}} P(x, y)\right]\right\} \\
+ & I_{y}^{v_{2}}\left\{D_{x}^{v_{1}-v_{2}} Q(x, y)\right. \\
& \left.C^{-}-D_{x}^{v_{1}-v_{2}}\left[Q(x, y)-D_{x}^{-v_{1}} D_{x}^{v_{1}} Q(x, y)\right]\right\} \\
= & { }_{x_{0}}^{x_{1}} I_{x}^{v_{2}}\left\{D_{y}^{v_{1}-v_{2}} P-D_{y}^{v_{1}-v_{2}}\left[P-D_{y}^{-v_{1}} D_{y}^{v_{1}} P\right]\right\} \\
+ & { }_{x_{1}}^{x_{1}} I_{x}^{v_{2}}\left\{D_{y}^{v_{1}-v_{2}} P-D_{y}^{v_{1}-v_{2}}\left[P-D_{y}^{-v_{1}} D_{y}^{v_{1}} P\right]\right\} \\
+ & +{ }_{y_{0}}^{y_{1}} I_{y}^{v_{2}}\left\{D_{x}^{v_{1}-v_{2}} Q-D_{x}^{v_{1}-v_{2}}\left[Q-D_{x}^{-v_{1}} D_{x}^{v_{1}} Q\right]\right\} \\
+ & +{ }_{y_{1}}^{y_{1}} I_{y}^{v_{2}}\left\{D_{x}^{v_{1}-v_{2}} Q-D_{x}^{v_{1}-v_{2}}\left[Q-D_{x}^{-v_{1}} D_{x}^{v_{1}} Q\right]\right\} \equiv 0 .
\end{aligned}
$$

Since $\sum_{m=0}^{\infty} \sum_{n=0}^{m} \equiv \sum_{n=0}^{\infty} \sum_{m=n}^{\infty}$ and $\left(\begin{array}{c}v \\ r+n\end{array}\right)\left(\begin{array}{c}r+n \\ n\end{array}\right) \equiv\left(\begin{array}{c}v \\ n\end{array}\right)\left(\begin{array}{c}v-n \\ r\end{array}\right)$, we have

$$
D_{x-a}^{v}(f g)=\sum_{n=0}^{\infty}\left[\left(\begin{array}{l}
v \\
n
\end{array}\right)\left(D_{x-a}^{v-n} f\right) D_{x-a}^{n} g\right]
$$

Referring to the homogeneous properties of fractional calculus and (10), we can derive that $\left(I_{x}^{v_{2}} I_{y}^{v_{2}}\right)_{\Omega}\left[D_{x}^{v_{1}}\left(u \varphi_{x}\right)+\right.$
$\left.D_{y}^{v_{1}}\left(u \varphi_{y}\right)\right]=\left(I_{x}^{v_{2}} I_{y}^{v_{2}}\right)_{\Omega}\left[D_{x}^{v_{1}}\left(u \varphi_{x}\right)-D_{y}^{v_{1}}\left(-u \varphi_{y}\right)\right]=0$. Then, we have

$$
\begin{aligned}
& \left(I_{x}^{v_{2}} I_{y}^{v_{2}}\right)_{\Omega} D^{v_{1}} u \cdot \vec{\varphi} \\
& =\left(I_{x}^{v_{2}} I_{y}^{v_{2}}\right)_{\Omega}\left[\left(D_{x}^{v_{1}} u\right) \varphi_{x}+\left(D_{y}^{v_{1}} u\right) \varphi_{y}\right] \\
& =-\left(I_{x}^{v_{2}} I_{y}^{v_{2}}\right)_{\Omega} \sum_{n=1}^{\infty}\left(\begin{array}{c}
v_{1} \\
n
\end{array}\right)\left[\left(D_{x}^{v_{1}-n} u\right) D_{x}^{n} \varphi_{x}+\left(D_{y}^{v_{1}-n} u\right) D_{y}^{n} \varphi_{y}\right],
\end{aligned}
$$

where the sign - denotes the inner product. Similar to the definition of fractional-order divergence operator $\operatorname{div}^{v} \vec{\varphi}=$ $D^{v} \cdot \vec{\varphi}=D_{x}^{v} \varphi_{x}+D_{y}^{v} \varphi_{y}$, we consider the $v$-order fractional differential operator to be $P^{v}=\sum_{n=1}^{\infty}\left(\begin{array}{c}v \\ n\end{array}\right)\left[i\left(\left(D_{x}^{v-n} u\right) / u\right) D_{x}^{n}+\right.$ $\left.j\left(\left(D_{y}^{v-n} u\right) / u\right) D_{y}^{n}\right]$ and the $v$-order fractional divergence operator to be $\operatorname{div} P^{v} \vec{\varphi}=P^{v} \cdot \vec{\varphi}=P_{x}^{v} \varphi_{x}+P_{y}^{v} \varphi_{y}$, where both $\operatorname{div} P^{v}$ and $P^{v}$ are the linear operators. In light of Hilbert adjoint operator theory [85] and (12), we can derive

$$
\begin{aligned}
\left(I_{x}^{v_{2}} I_{y}^{v_{2}}\right)_{\Omega} D^{v_{1}} u \cdot \vec{\varphi} & =\left\langle D^{v_{1}} u, \vec{\varphi}\right\rangle^{v_{2}}=\left\langle u,\left(D^{v_{1}}\right)^{*} \vec{\varphi}\right\rangle^{v_{2}} \\
& =\left(I_{x}^{v_{2}} I_{y}^{v_{2}}\right)_{\Omega} u\left(\left(D^{v_{1}}\right)^{*} \vec{\varphi}\right) \\
& =-\left(I_{x}^{v_{2}} I_{y}^{v_{2}}\right)_{\Omega} u\left(P^{v_{1}} \cdot \vec{\varphi}\right) \\
& =-\left(I_{x}^{v_{1}} I_{y}^{v_{2}}\right)_{\Omega} u\left(\operatorname{div} P^{v_{1}} \vec{\varphi}\right),
\end{aligned}
$$

where $\langle,\rangle^{v_{2}}$ denotes the integral form of the $v_{2}$-order fractional inner product. $\left(D^{v}\right)^{*}$ is $v$-order fractional Hilbert adjoint operator of $D^{v}$. Then, it follows that

$$
\left(D^{v}\right)^{*}=-\operatorname{div} P^{v},
$$

where $\left(D^{v}\right)^{*}$ is a fractional Hilbert adjoint operator. When $v_{1}=v_{2}=1$, (12) becomes

$$
\begin{aligned}
\left(I_{x}^{1} I_{y}^{1}\right)_{\Omega} D^{1} u \cdot \vec{\varphi} & =\left\langle D^{1} u, \vec{\varphi}\right\rangle^{1}=\left\langle u,\left(D^{1}\right)^{*} \vec{\varphi}\right\rangle^{1} \\
& =\left(I_{x}^{1} I_{y}^{1}\right)_{\Omega} u\left(\left(D^{1}\right)^{*} \vec{\varphi}\right)=-I_{x}^{1} I_{y}^{1} u\left(\operatorname{div}^{1} \vec{\varphi}\right),
\end{aligned}
$$

where $\langle,\rangle^{1}$ denotes the integral form of the first-order inner product, $\operatorname{div}^{1} \vec{\varphi}=D^{1} \bullet \vec{\varphi}=D_{x}^{1} \varphi_{x}+D_{y}^{1} \varphi_{y}$ represents the first-order divergence operator, and $\left(D^{1}\right)^{*}$ represents the first-order Hilbert adjoint operator of $D^{1}$. As for digital image, we find that

$$
\left(D^{1}\right)^{*}=-\operatorname{div}^{1} .
$$


Equations (14) and (16) have shown that the first-order Hilbert adjoint operator is the special case of that of fractional order. When $\left(I_{x}^{v_{2}} I_{y}^{v_{2}}\right)_{\Omega} D^{v_{1}} u \cdot \vec{\varphi}=0$, (14) becomes

$$
\begin{aligned}
& \left(I_{x}^{v_{2}} I_{y}^{v_{2}}\right)_{\Omega} D^{v_{1}} u \cdot \vec{\varphi} \\
& =-\left(I_{x}^{v_{2}} I_{y}^{v_{2}}\right)_{\Omega} \sum_{n=1}^{\infty}\left(\begin{array}{c}
v_{1} \\
n
\end{array}\right)\left[\left(D_{x}^{v_{1}-n} u\right) D_{x}^{n} \varphi_{x}+\left(D_{y}^{v_{1}-n} u\right) D_{y}^{n} \varphi_{y}\right] \\
& =0
\end{aligned}
$$

Since the $x$ line and $y$ line meet at right angle, it has $\left(D_{x}^{v_{1}-n} u\right) D_{x}^{n} \varphi_{x}+\left(D_{y}^{v_{1}-n} u\right) D_{y}^{n} \varphi_{y}=\left(D_{x}^{v_{1}-n} u, D_{y}^{v_{1}-n} u\right) \cdot\left(D_{x}^{n} \varphi_{x}\right.$, $\left.D_{y}^{n} \varphi_{y}\right)$. As for any two-dimensional function $u$, the corresponding $D_{x}^{v_{1}-n} u$ and $D_{y}^{v_{1}-n} u$ are randomly chosen. According to the fundamental lemma of calculus of variations [83], we know that $\left(D_{x}^{n} \varphi_{x}, D_{y}^{n} \varphi_{y}\right)=(0,0)$ is required to make (17) established. Since $n$ is the positive integer belonging to $1 \rightarrow \infty$, we only need $\left(D_{x}^{1} \varphi_{x}\right.$, $\left.D_{y}^{1} \varphi_{y}\right)=(0,0)$ to make $\left(D_{x}^{n} \varphi_{x}, D_{y}^{n} \varphi_{y}\right)=(0,0)$ established. Equation (17) can be rewritten as $-\left(I_{x}^{v_{2}} I_{y}^{v_{2}}\right)_{\Omega}\left(\begin{array}{c}v_{1} \\ 1\end{array}\right)\left[\left(D_{x}^{v_{1}-1} u\right) D_{x}^{1} \varphi_{x}+\left(D_{y}^{v_{1}-1} u\right) D_{y}^{1} \varphi_{y}\right]=$ $-\left(I_{x}^{v_{2}} I_{y}^{v_{2}}\right)_{\Omega}\left(\begin{array}{c}v_{1} \\ 1\end{array}\right)\left(D_{x}^{v_{1}-1} u, D_{y}^{v_{1}-1} u\right) \cdot\left(D_{x}^{1} \varphi_{x}, D_{y}^{1} \varphi_{y}\right)=0$. When and only when the below equation is satisfied, (17) is established:

$$
-\left(\begin{array}{c}
v_{1} \\
1
\end{array}\right)\left[D_{x}^{1} \varphi_{x}+D_{y}^{1} \varphi_{y}\right]=\frac{\Gamma\left(1-v_{1}\right)}{\Gamma\left(-v_{1}\right)}\left[D_{x}^{1} \varphi_{x}+D_{y}^{1} \varphi_{y}\right]=0 \text {. }
$$

Equation (18) is the fractional Euler-Lagrange formula, which corresponds to $\left(I_{x}^{v_{2}} I_{y}^{v_{2}}\right)_{\Omega} D^{v_{1}} u \cdot \vec{\varphi}=0$.

Also, if one considers $\Phi_{1}\left(D^{v_{1}} u\right)$ to be the numerical function of vector function $D^{v_{1}} u$ and $\Phi_{2}(\vec{\varphi})$ to be the numerical function of differintegrable vector function $\vec{\varphi}(x, y)=i \varphi_{x}+$ $j \varphi_{y}[1-6]$, similarly, when $\left(I_{x}^{v_{2}} I_{y}^{v_{2}}\right)_{\Omega} \Phi_{1}\left(D^{v_{1}} u\right) \Phi_{2}(\vec{\varphi}) D^{v_{1}} u \cdot \vec{\varphi}=$ 0 , (14) can be written as

$$
\begin{aligned}
&\left(I_{x}^{v_{2}} I_{y}^{v_{2}}\right)_{\Omega} \Phi_{1}\left(D^{v_{1}} u\right) \Phi_{2}(\vec{\varphi}) D^{v_{1}} u \cdot \vec{\varphi} \\
&=\left(I_{x}^{v_{2}} I_{y}^{v_{2}}\right)_{\Omega} \Phi_{1}\left(D^{v_{1}} u\right) D^{v_{1}} u \cdot\left(\Phi_{2}(\vec{\varphi}) \vec{\varphi}\right) \\
&=-\left(I_{x}^{v_{2}} I_{y}^{v_{2}}\right)_{\Omega} \sum_{n=1}^{\infty}\left(\begin{array}{c}
v_{1} \\
n
\end{array}\right) {\left[\left(\Phi_{1}\left(D^{v_{1}} u\right) D_{x}^{v_{1}-n} u\right)\right.} \\
& \times D_{x}^{n}\left(\Phi_{2}(\vec{\varphi}) \varphi_{x}\right) \\
&+\left(\Phi_{1}\left(D^{v_{1}} u\right) D_{y}^{v_{1}-n} u\right) \\
&\left.\times D_{y}^{n}\left(\Phi_{2}(\vec{\varphi}) \varphi_{y}\right)\right]=0
\end{aligned}
$$

Equation (19) is established, when and only when

$$
\begin{gathered}
-\left(\begin{array}{c}
v_{1} \\
1
\end{array}\right)\left[D_{x}^{1}\left(\Phi_{2}(\vec{\varphi}) \varphi_{x}\right)+D_{y}^{1}\left(\Phi_{2}(\vec{\varphi}) \varphi_{y}\right)\right] \\
=\frac{\Gamma\left(1-v_{1}\right)}{\Gamma\left(-v_{1}\right)}\left[D_{x}^{1}\left(\Phi_{2}(\vec{\varphi}) \varphi_{x}\right)\right. \\
\left.+D_{y}^{1}\left(\Phi_{2}(\vec{\varphi}) \varphi_{y}\right)\right]=0 .
\end{gathered}
$$

Equation (20) is the fractional Euler-Lagrange formula corresponding to $\left(I_{x}^{v_{2}} I_{y}^{v_{2}}\right) \Phi_{1}\left(D^{v_{1}} u\right) \Phi_{2}(\vec{\varphi}) D^{v_{1}} u \cdot \vec{\varphi}=0$.

Since it has $D_{x-a}^{v}[0] \equiv 0$ no matter what $v$ is, we know that the fractional Euler-Lagrange formulas (18) and (20) are irrelevant to the integral order $v_{2}$ of fractional surface integral $\left(I_{x}^{v_{2}} I_{y}^{v_{2}}\right)_{\Omega}$. We therefore only adopt the first-order surface integral $\left(I_{x}^{v} I_{y}^{v}\right)_{\Omega}$ instead of that of fractional order, when we discuss the energy norm of fractional partial differential equation-based model for texture denoising below.

3.3. The Fractional Partial Differential Equation-Based Denoising Approach for Texture Image. Based on the fractional Euler-Lagrange formula for two-dimensional image, we can implement a fractional partial differential equation-based denoising model for texture image.

$s(x, y)$ represents the gray value of the pixel $(x, y)$, where $\Omega \subset R^{2}$ is the image region, that is, $(x, y) \in \Omega$. Consider $s(x, y)$ to be the noised image and $s_{0}(x, y)$ to represent the desired clean image. Since the noise can be converted to additive noise by log processing when it is multiplicative noise and to additive noise by frequency transform and log processing when it is convolutive noise, we assume that $n(x, y)$ is additive noise, that is, $s(x, y)=s_{0}(x, y)+n(x, y)$ without loss of generality. Consider $n(x, y)$ to represent the additive noise, that is, $s(x, y)=s_{0}(x, y)+n(x, y)$; we adopted the fractional extreme to form the energy norm. Similar to the fractional $\delta$-cover in the Hausdorff measure $[96,97]$, we consider the fractional total variation of image $s$ to be $\left|D^{v_{1}} s\right|^{v_{2}}=\left(\sqrt{\left(D_{x}^{v_{1}} s\right)^{2}+\left(D_{y}^{v_{1}} s\right)^{2}}\right)^{v_{2}}$, where $v_{2}$ is any real number including fractional number and $\left|D^{v_{1}} s\right|^{v_{2}}$ is the hypercube measure. We assume the fractional variationbased fractional total variation as

$$
E_{\mathrm{FTV}}(s)=\left(I_{x}^{1} I_{y}^{1}\right)_{\Omega}\left[f\left(\left|D^{v_{1}} s\right|^{v_{2}}\right)\right]=\iint_{\Omega} f\left(\left|D^{v_{1}} s\right|^{v_{2}}\right) d x d y .
$$

Consider $s$ to be the $v_{3}$-order extremal surface of $E_{\mathrm{FTV}}$, the test function is the admitting curve surface close to the extremal surface, that is, $\xi(x, y) \in C_{0}^{\infty}(\Omega)$, we then correlate and merge $s$ and $\xi$ by $s+(\beta-1) \xi$. When $\beta=1$, it is the $v_{3}$-order extremal surface $s$. Assume that $\Psi_{1}(\beta)=E_{\mathrm{FTV}}[s+(\beta-1) \xi]$ and $\Psi_{2}(\beta)=\iint_{\Omega} \lambda\left[s+(\beta-1) \xi-s_{0}\right] s_{0} d x d y$, where $\Psi_{2}(\beta)$ is the cross energy of the noise and clean signal $s_{0}$, that is, $\left[s+(\beta-1) \xi-s_{0}\right]$, and it also the measurement of the similarity between $\left[s+(\beta-1) \xi-s_{0}\right]$ and $s_{0}$. We therefore can explain the anisotropic diffusion as energy dissipation process for solving the $v_{3}$-order minimum of fractional energy norm $E_{\mathrm{FTV}}$, that is, the process for solving minimum of $\Psi_{2}(\beta)$ is to obtain the minimum similarity between the noise and the clean signal. Here, $\Psi_{2}(\beta)$ plays the role of nonlinear fidelity during denoising, and $\lambda$ is regularized parameter. Fractional total variation-based fractional energy norm in surface family $s+(\beta-1) \xi$ can be expressed by

$$
\begin{aligned}
\Psi(\beta)= & \Psi_{1}(\beta)+\Psi_{2}(\beta) \\
=\left(I_{x}^{1} I_{y}^{1}\right)_{\Omega}[ & f\left(\left|D^{v_{1}} s+(\beta-1) D^{v_{1}} \xi\right|^{v_{2}}\right) \\
& \left.+\lambda\left[s+(\beta-1) \xi-s_{0}\right] s_{0}\right]
\end{aligned}
$$




$$
\begin{aligned}
=\iint_{\Omega}[ & f\left(\left|D^{v_{1}} s+(\beta-1) D^{v_{1}} \xi\right|^{v_{2}}\right) \\
& \left.+\lambda\left[s+(\beta-1) \xi-s_{0}\right] s_{0}\right] d x d y .
\end{aligned}
$$

As for $\Psi_{1}(\beta)$, if $v_{3}$-order fractional derivative of $f\left(\left|D^{v_{1}} s+(\beta-1) D^{v_{1}} \xi\right|^{v_{2}}\right)$ exists, $\Psi_{1}(\beta)$ has the $v_{3}$-order fractional minimum or stationary point when $\beta=1$. Referring to the linear properties of fractional differential operator, we have

$$
\begin{aligned}
\left.D_{\beta}^{v_{3}} \Psi_{1}(\beta)\right|_{\beta=1} & =\left.\frac{\partial^{v_{3}}}{\partial \beta^{v_{3}}} \iint_{\Omega} f\left(|\vec{\varphi}|^{v_{2}}\right) d x d y\right|_{\beta=1} \\
& =\left.\iint_{\Omega} \frac{\partial^{v_{3}}}{\partial \beta^{v_{3}}} f\left(|\vec{\varphi}|^{v_{2}}\right) d x d y\right|_{\beta=1}=0
\end{aligned}
$$

where it has $\vec{\varphi}^{2}=|\vec{\varphi}|^{2}=\left[{\sqrt{(\vec{\varphi})^{2}}}^{2}=\vec{\varphi} \bullet \vec{\varphi}\right.$ as for the vector $\vec{\varphi}=$ $D^{v_{1}} s+(\beta-1) D^{v_{1}} \xi$, and the signal $\bullet$ denotes the inner product. Unlike the traditional first-order variation, $(23)$ is the $v_{3}$ order fractional extreme of $\Psi_{1}(\beta)$, which aims to nonlinearly preserve the complex texture details as much as possible when denoising by using the special properties of fractional calculus that it can nonlinearly maintain the low-frequency contour feature in the smooth area to the furthest degree and nonlinearly enhance the high-frequency edge information in those areas where gray level changes frequently and also nonlinearly enhance the high-frequency texture details in those areas where gray level does not change obviously [3338].

Provided that $v$ is a fractional number, when $n>v$, it has $\left(\begin{array}{l}v \\ n\end{array}\right)=(-1)^{n} \Gamma(n-v) / \Gamma(-v) \Gamma(n+1) \neq 0$. Referring to (11) and Faà de Bruno formula [95], we can derive the rule of fractional calculus of composite function as

$$
\begin{aligned}
D_{\beta-a}^{v} f[g(\beta)]= & \frac{(\beta-a)^{-v}}{\Gamma(1-v)} f \\
& +\sum_{n=1}^{\infty}\left(\begin{array}{l}
v \\
n
\end{array}\right) \frac{(\beta-a)^{n-v}}{\Gamma(n-v+1)} n ! \\
& \times \sum_{m=1}^{n} D_{g}^{m} f \sum \prod_{k=1}^{n} \frac{1}{P_{k} !}\left[\frac{D_{\beta-a}^{k} g}{k !}\right]^{P_{k}},
\end{aligned}
$$

where $g(\beta)=|\vec{\varphi}|^{v_{2}}$ and $a$ is the constant. $n=0$ is separated from summation item. From (24), we know that the fractional derivative of composite function is the summation of infinite items. Here, $P_{k}$ satisfies

$$
\sum_{k=1}^{n} k P_{k}=n, \quad \sum_{k=1}^{n} P_{k}=m .
$$

The third signal $\sum$ in (25) denotes the summation of $\left.\left\{\prod_{k=1}^{n}\left(1 / P_{k} !\right)\left[D_{\beta-a}^{k} g / k !\right]^{P_{k}}\right\}\right|_{m=1 \rightarrow n}$ of the combination of
$\left.P_{k}\right|_{m=1 \rightarrow n}$ that satisfied (25). Recalling (23), (24) and the property of Gamma function, we can derive

$$
\begin{aligned}
& \left.\iint_{\Omega} \frac{\partial^{v_{3}}}{\partial \beta^{v_{3}}} f\left(|\vec{\varphi}|^{v_{2}}\right) d x d y\right|_{\beta=1} \\
& =\iint_{\Omega} \frac{\beta^{-v_{3}} f\left(|\vec{\varphi}|^{v_{2}}\right)}{\Gamma\left(1-v_{3}\right)}+\sum_{n=1}^{\infty}\left(\begin{array}{c}
v_{3} \\
n
\end{array}\right) \frac{\beta^{n-v_{3}}}{\Gamma\left(n-v_{3}+1\right)} n ! \\
& \quad \times\left.\sum_{m=1}^{n} D_{|\vec{\varphi}|^{v_{2}}}^{m} f \sum \prod_{k=1}^{n} \frac{1}{P_{k} !}\left[\frac{D_{\beta}^{k}\left(|\vec{\varphi}|^{v_{2}}\right)}{k !}\right]^{P_{k}} d x d y\right|_{\beta=1} \\
& =\iint_{\Omega} \frac{f\left(\left|D^{v_{1}} s\right|^{v_{2}}\right)}{\Gamma\left(1-v_{3}\right)}+\sum_{n=1}^{\infty} \frac{(-1)^{n} \Gamma\left(n-v_{3}\right)}{\Gamma\left(-v_{3}\right) \Gamma\left(n-v_{3}+1\right)} \\
& \quad \times\left.\sum_{m=1}^{n} D_{|\vec{\varphi}|^{v_{2}}}^{m} f\right|_{\beta=1} \sum_{k=1}^{n} \frac{1}{P_{k} !}\left[\frac{\left.D_{\beta}^{k}\left(|\vec{\varphi}|^{v_{2}}\right)\right|_{\beta=1}}{k !}\right]^{P_{k}} \\
& \quad \times d x d y=0 .
\end{aligned}
$$

Without loss of the generality, we consider $f(\eta)=\eta$ for simple calculation; it then has $D_{\eta}^{1} f(\eta)=1$ and $D_{\eta}^{m} f(\eta) \stackrel{m \geq 2}{=} 0$; thus (26) can be reduced as

$$
\begin{aligned}
& \iint_{\Omega} \frac{\left|D^{v_{1}} s\right|^{v_{2}}}{\Gamma\left(1-v_{3}\right)}+\sum_{n=1}^{\infty} \frac{(-1)^{n} \Gamma\left(n-v_{3}\right)}{\Gamma\left(-v_{3}\right) \Gamma\left(n-v_{3}+1\right)} \\
& \quad \times\left\{\prod_{k=1}^{n} \frac{1}{P_{k} !}\left[\frac{\left.D_{\beta}^{k}\left(|\vec{\varphi}|^{v_{2}}\right)\right|_{\beta=1}}{k !}\right]^{P_{k}}\right\}_{m=1} d x d y=0 .
\end{aligned}
$$

We know from (25), when $m=1, P_{k}$ satisfies $P_{n}=1$ and $P_{1}=P_{2}=\cdots=P_{n-1}=0$; then it can be furtherly reduced as

$$
\begin{gathered}
\iint_{\Omega} \frac{\left|D^{v_{1}} s\right|^{v_{2}}}{\Gamma\left(1-v_{3}\right)}+\sum_{n=1}^{\infty} \frac{(-1)^{n} \Gamma\left(n-v_{3}\right)}{\Gamma\left(-v_{3}\right) \Gamma\left(n-v_{3}+1\right)} \\
\times \frac{\left.D_{\beta}^{n}\left(|\vec{\varphi}|^{v_{2}}\right)\right|_{\beta=1}}{n !} d x d y=0 .
\end{gathered}
$$

When $n$ takes odd number $(n=2 k+1, k=0,1,2,3, \ldots)$ and even number $(n=2 k, k=1,2,3, \ldots)$, respectively, the expressions of $\left.D_{\beta}^{n}\left(|\vec{\varphi}|^{v_{2}}\right)\right|_{\beta=1}$ are also different:

$$
\begin{aligned}
&\left.D_{\beta}^{n}\left(|\vec{\varphi}|^{v_{2}}\right)\right|_{\beta=1} ^{n=2 k+1}= \prod_{\tau=1}^{n}\left(v_{2}-\tau+1\right)\left|D^{v_{1}} s\right|^{v_{2}-n-1} \\
& \times\left|D^{v_{1}} \xi\right|^{n-1}\left(D^{v_{1}} \xi\right) \cdot\left(D^{v_{1}} s\right), \\
&\left.D_{\beta}^{n}\left(|\vec{\varphi}|^{v_{2}}\right)\right|_{\beta=1} ^{n=2 k}=\prod_{\tau=1}^{n}\left(v_{2}-\tau+1\right)\left|D^{v_{1}} s\right|^{v_{2}-n}\left|D^{v_{1}} \xi\right|^{n} .
\end{aligned}
$$


Put (29) into (28), then it becomes

$$
\begin{aligned}
& \iint_{\Omega} \sum_{k=0}^{\infty} \frac{\prod_{\tau=1}^{2 k}\left(v_{2}-\tau+1\right)\left|D^{v_{1}} s\right|^{v_{2}-2 k-2}\left|D^{v_{1}} \xi\right|^{2 k}}{\Gamma\left(-v_{3}\right)(2 k) !}\left(D^{v_{1}} s\right) \\
& \quad D^{v_{1}}\left[\frac{\Gamma\left(2 k-v_{3}\right)}{\Gamma\left(2 k-v_{3}+1\right)} s-\frac{\left(v_{2}-2 k\right) \Gamma\left(2 k-v_{3}+1\right)}{(2 k+1) \Gamma\left(2 k-v_{3}+2\right)} \xi\right] \\
& \quad \times d x d y=0 .
\end{aligned}
$$

We consider that $\prod_{\tau=1}^{n}\left(v_{2}-\tau+1\right) \stackrel{n=0}{=} 1$. Since the corresponding $D^{v_{1}} \xi$ is randomly chosen for any two-dimensional numerical function $\xi, D^{v_{1}}\left[\left(\Gamma\left(2 k-v_{3}\right) / \Gamma\left(2 k-v_{3}+1\right)\right) s-\left(\left(v_{2}-\right.\right.\right.$ $\left.\left.2 k) \Gamma\left(2 k-v_{3}+1\right) /(2 k+1) \Gamma\left(2 k-v_{3}+2\right) \xi\right)\right]$ is random. Referring to (20), the corresponding fractional Euler-Lagrange formula of $(30)$ is

$$
\begin{aligned}
& \frac{\Gamma\left(1-v_{1}\right)}{\Gamma\left(-v_{1}\right) \Gamma\left(-v_{3}\right)} \sum_{k=0}^{\infty} \frac{\prod_{\tau=1}^{2 k}\left(v_{2}-\tau+1\right)}{(2 k) !} \\
& \times\left[D_{x}^{1}\left(\left|D^{v_{1}} s\right|^{v_{2}-2 k-2} D_{x}^{v_{1}} s\right)+D_{y}^{1}\left(\left|D^{v_{1}} s\right|^{v_{2}-2 k-2} D_{y}^{v_{1}} s\right)\right]=0,
\end{aligned}
$$

where it has $\prod_{\tau=1}^{n}\left(v_{2}-\tau+1\right) \stackrel{n=0}{=} 1$. It is the corresponding fractional Euler-Lagrange formula of (23).

As for $\Psi_{2}(\beta)$, it has

$$
\begin{aligned}
& \left.D_{\beta}^{v_{3}} \Psi_{2}(\beta)\right|_{\beta=1} \\
& \quad=\left.D_{\beta}^{v_{3}}\left(I_{x}^{1} I_{y}^{1}\right)_{\Omega} \lambda\left[s+(\beta-1) \xi-s_{0}\right] s_{0}\right|_{\beta=1} \\
& \quad=\iint_{\Omega} \frac{\lambda s_{0}}{\Gamma\left(1-v_{3}\right) \Gamma\left(2-v_{3}\right)} \\
& \quad \times\left[\Gamma\left(2-v_{3}\right)\left(s-s_{0}-\xi\right)+\Gamma\left(1-v_{3}\right) \xi\right] d x d y=0 .
\end{aligned}
$$

Since the test function $\xi$ is randomly chosen, $\Gamma\left(2-v_{3}\right)\left(s-s_{0}-\right.$ $\xi)+\Gamma\left(1-v_{3}\right) \xi$ is also random. According to the fundamental lemma of calculus of variations [83], we know that to make (32) established, it must have

$$
\frac{\lambda s_{0}}{\Gamma\left(1-v_{3}\right) \Gamma\left(2-v_{3}\right)}=0 .
$$

Equations (23) and (32), respectively, are the $v_{3}$-order minimal value of $\Psi_{1}(\beta)$ and $\Psi_{1}(\beta)$. Thus, when we take $v=v_{3} \neq 1$, 2 , and 3 , the $v_{3}$-order minimal value of (22) can be expressed by

$$
\begin{aligned}
\frac{\partial^{v_{3}} s}{\partial t^{v_{3}}}= & \frac{-\Gamma\left(1-v_{1}\right)}{\Gamma\left(-v_{1}\right) \Gamma\left(-v_{3}\right)} \\
& \times \sum_{k=0}^{\infty} \frac{\prod_{\tau=1}^{2 k}\left(v_{2}-\tau+1\right)}{(2 k) !}
\end{aligned}
$$

$$
\begin{gathered}
\times\left[D_{x}^{1}\left(\left|D^{v_{1}} s\right|^{v_{2}-2 k-2} D_{x}^{v_{1}} s\right)\right. \\
\left.+D_{y}^{1}\left(\left|D^{v_{1}} s\right|^{v_{2}-2 k-2} D_{y}^{v_{1}} s\right)\right] \\
-\frac{\lambda s_{0}}{\Gamma\left(1-v_{3}\right) \Gamma\left(2-v_{3}\right)}
\end{gathered}
$$

where it has $\prod_{\tau=1}^{n}\left(v_{2}-\tau+1\right) \stackrel{n=0}{=} 1 . \partial^{v_{3}} s / \partial t^{v_{3}}$ is calculated by the approach of fractional difference. We must compute $\lambda(\mathrm{t})$. If image noise $n(x, y)$ is the white noise, it has $\iint_{\Omega} n(x, y) d x d y=\iint_{\Omega}\left(s-s_{0}\right) d x d y=0$. When $\partial^{v_{3}} s / \partial t^{v_{3}}=0$, it converges to a stable state. Then, we merely multiply $\left(s-s_{0}\right)^{2}$ at both sides of (34) and integrate by parts over $\Omega$ and the left side of (34) vanishes:

$$
\begin{gathered}
\lambda(t)=\frac{-\Gamma\left(1-v_{1}\right) \Gamma\left(1-v_{3}\right) \Gamma\left(2-v_{3}\right)}{\sigma^{2} \Gamma\left(-v_{1}\right) \Gamma\left(-v_{3}\right) s_{0}} \\
\times \iint_{\Omega} \sum_{k=0}^{\infty} \frac{\prod_{\tau=1}^{2 k}\left(v_{2}-\tau+1\right)}{(2 k) !} \\
\times\left[D_{x}^{1}\left(\left|D^{v_{1}} s\right|^{v_{2}-2 k-2} D_{x}^{v_{1}} s\right)\right. \\
\left.\quad+D_{y}^{1}\left(\left|D^{v_{1}} s\right|^{v_{2}-2 k-2} D_{y}^{v_{1}} s\right)\right] \\
\times\left(s-s_{0}\right)^{2} d x d y .
\end{gathered}
$$

Here, we simply note the fractional partial differential equation-based denoising model as FDM (a fractional developmental mathematics-based approach for texture image denoising). When numerical iterating, we need to perform low-pass filtering to completely remove the faint noise in very low-frequency and direct current. We know from (34) and (35) that FDM enhances the nonlinear regulation effects of order $v_{2}$ by continually multiplying function $\prod_{\tau=1}^{2 k}\left(v_{2}-\tau+1\right)$ and power $v_{2}-2 k-2$ of $\left|D^{v_{1}} s\right|$ and enhance the nonlinear regulation effects of order $v_{3}$ by increasing $\Gamma\left(-v_{3}\right)$ in the denominator. Also, we know from (34) that FDM is the traditional potential equation or elliptic equation when $v_{3}=0$, the traditional heat conduction equation or parabolic equation when $v_{3}=1$, and the traditional wave equation or hyperbolic equation when $v_{3}=2$. FDM is the continuous interpolation of traditional potential equation and heat conduction equation when $0<v_{3}<1$ and the continuous interpolation of traditional heat conduction equation and wave equation when $1<v_{3}<2$. FDM has pushed the traditional integerorder partial differential equation-based image processing approach from the anisotropic diffusion of integer-order heat conduction equation to that of fractional partial differential equation in the mathematical and physical sense.

\section{Experiments and Theoretical Analyzing}

4.1. Numerical Implementation of the Fractional Partial Differential Equation-Based Denoising Model for Texture Image. We know from (34) and (35) that we should obtain 


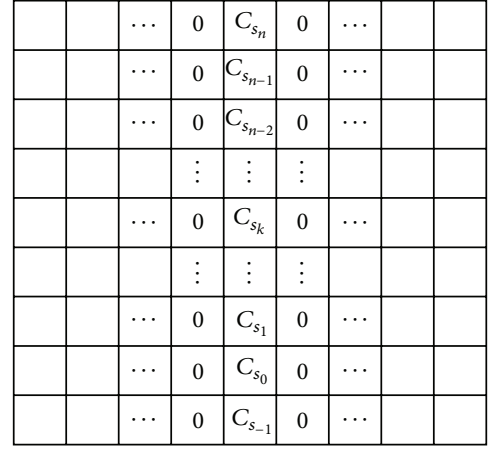

(a)

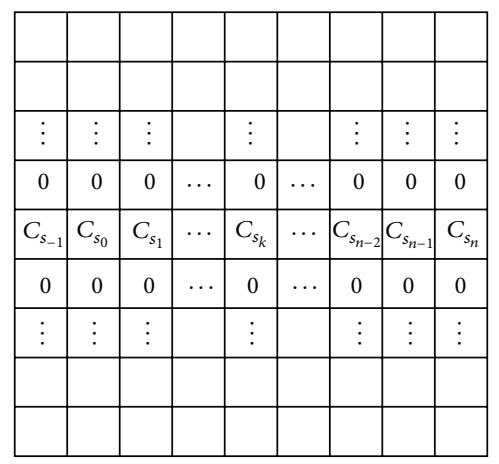

(d)

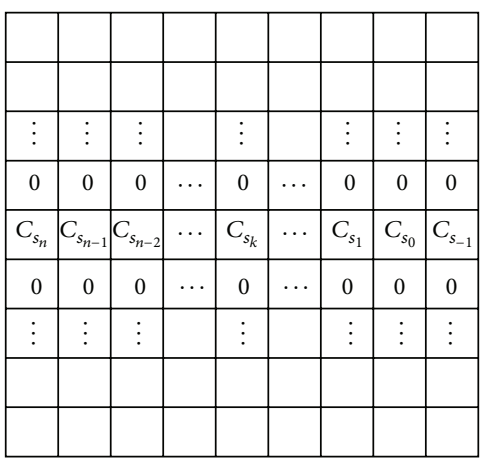

(b)

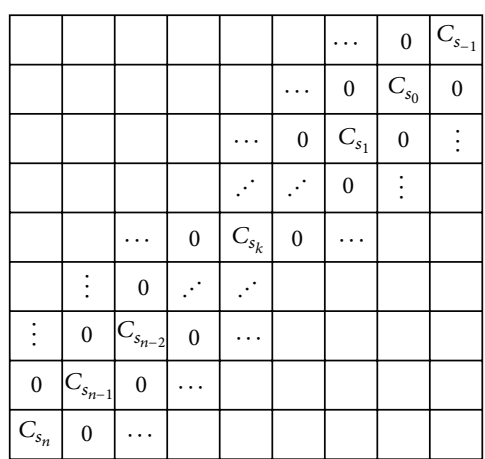

(e)

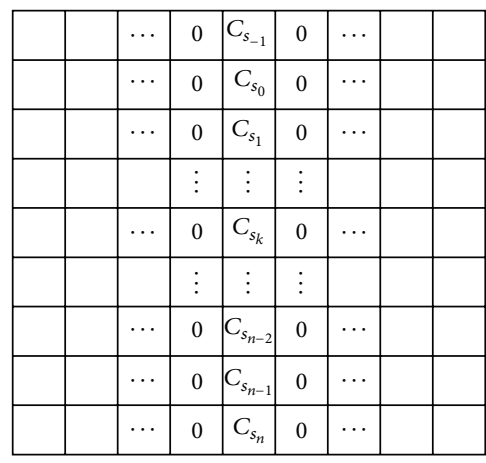

(c)

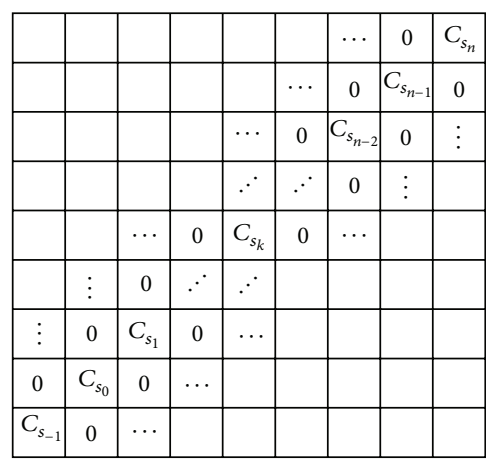

(f)

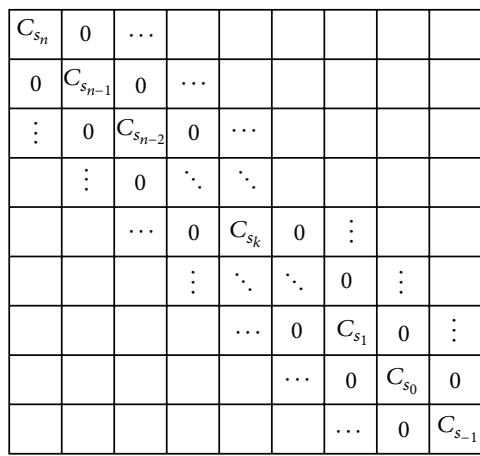

(g)

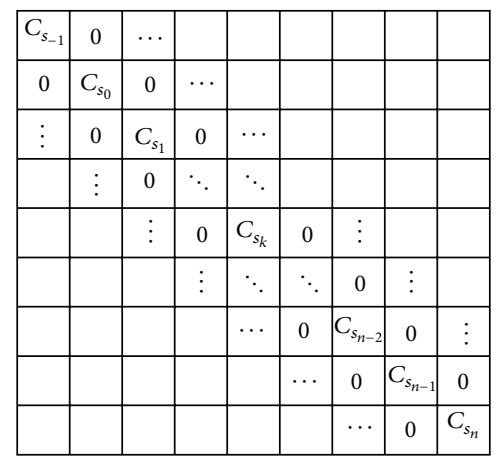

(h)

FIGURE 3: Fractional differential masks $D^{v}$, respectively, on the eight directions. (a) Fractional differential operator on $x$-coordinate negative direction, noted as $D_{x-}^{v}$. (b) Fractional differential operator on $y$-coordinate negative direction, noted as $D_{y-}^{v}$. (c) Fractional differential operator on $x$-coordinate positive direction, noted as $D_{x+}^{v}$. (d) Fractional differential operator on $y$-coordinate positive direction, noted as $D_{y+}^{v}$. (e) Fractional differential operator on left downward diagonal, noted as $D_{\text {ldd }}^{v}$. (f) Fractional differential operator on right upward diagonal, noted as $D_{\text {rud }}^{v}$ (g) Fractional differential operator on left upward diagonal, noted as $D_{\text {lud }}^{v}$. (h) Fractional differential operator on right downward diagonal, noted as $D_{\text {rdd }}^{v}$.

the fractional differential operator of two-dimensional digital image before implementing FDM. As for GrümwaldLetnikov definition of fractional calculus in (1), it may remove the limit symbol when $N$ is large enough; we then introduce the signal value at nonnode to the definition improving the convergence rate and accuracy, that is, $\left.\left(d^{v} / d x^{v}\right) s(x)\right|_{G-L} \cong$ $\left(x^{-v} N^{v} / \Gamma(-v)\right) \sum_{k=0}^{N-1}(\Gamma(k-v) / \Gamma(k+1)) s(x+(v x / 2 N)-$ $(k x / N))$. Using Lagrange 3-point interpolation equation to perform fractional interpolation when $v \neq 1$, we can obtain the fractional differential operators of YiFeiPU-2, respectively, on the eight symmetric directions [35, 38], which is shown in Figure 3.
In Figure 3, $C_{s_{-1}}$ is the coefficient of operator $s_{-1}=s(x+$ $x / N)$ at noncausal pixel, and $C_{s_{0}}$ is the coefficient of operator at pixel of interest $s_{0}=s(x) . n$ is often taken odd number. When $k \rightarrow n=2 m-1$, we can implement fractional differential operator of $(2 m+1) \times(2 m+1)$, whose coefficients of the operator, respectively, are $C_{s_{-1}}=(v / 4)+\left(v^{2} / 8\right), C_{s_{0}}=$ $1-\left(v^{2} / 2\right)-\left(v^{3} / 8\right), C_{s_{1}}=-(5 v / 4)+\left(5 v^{3} / 16\right)+\left(v^{4} / 16\right), \ldots$, $C_{s_{k}}=(1 / \Gamma(-v))\left[(\Gamma(k-v+1) /(k+1) !) \cdot\left((v / 4)+\left(v^{2} / 8\right)\right)+(\Gamma(k-\right.$ $\left.v) / k !) \cdot\left(1-\left(v^{2} / 4\right)\right)+(\Gamma(k-v-1) /(k-1) !) \cdot\left(-(v / 4)+\left(v^{2} / 8\right)\right)\right], \ldots$, $C_{s_{n-2}}=(1 / \Gamma(-v))\left[(\Gamma(n-v-1) /(n-1) !) \cdot\left((v / 4)+\left(v^{2} / 8\right)\right)+\right.$ $(\Gamma(n-v-2) /(n-2) !) \cdot\left(1-\left(v^{2} / 4\right)\right)+(\Gamma(n-v-3) /(n-3) !) \cdot$ 
$\left.\left(-(v / 4)+\left(v^{2} / 8\right)\right)\right], C_{s_{n-1}}=(\Gamma(n-v-1) /(n-1) ! \Gamma(-v))$. $\left(1-\left(v^{2} / 4\right)\right)+(\Gamma(n-v-2) /(n-2) ! \Gamma(-v)) \cdot\left(-(v / 4)+\left(v^{2} / 8\right)\right)$, and $C_{s_{n}}=(\Gamma(n-v-1) /(n-1) ! \Gamma(-v)) \cdot\left(-(v / 4)+\left(v^{2} / 8\right)\right)$ $[35,38]$. Spatial filtering of convolution operator is adopted as the numerical algorithm of fractional differential operator. We take the maximum value of fractional partial differential on the above 8 directions as the pixel's fractional calculus. As for fractional differential operator $D^{v}=i_{1} D_{x-}^{v}+i_{2} D_{y-}^{v}+$ $i_{3} D_{x+}^{v}+i_{4} D_{y+}^{v}+i_{5} D_{\text {ldd }}^{v}+i_{6} D_{\text {rud }}^{v}+i_{7} D_{\text {lud }}^{v}+i_{8} D_{\text {rdd }}^{v}$ in (34) and (35), when $v \neq 1 D_{x-}^{v}, D_{y-}^{v}, D_{x+}^{v}, D_{y+}^{v}, D_{\text {ldd }}^{v}, D_{\text {rud }}^{v}, D_{\text {lud }}^{v}$, $D_{\text {rdd }}^{v}$ can perform numerical computing referring to Figure 3 and their concerning coefficients $[35,38]$, and when $v=$ 1 , we take a special difference as the approximate of firstorder differential to maintain calculation stability, that is, $D_{x}^{1} s(x, y)=(2[s(x+1, y)-s(x-1, y)]+s(x+1, y+$ $1)-s(x-1, y+1)+s(x+1, y-1)-s(x-1, y-1)) / 4$, $D_{y}^{1} s(x, y)=(2[s(x, y+1)-s(x, y-1)]+s(x+1, y+1)-$ $s(x+1, y-1)+s(x-1, y+1)-s(x-1, y-1)) / 4$.

If time equal interval is $\Delta t$, time $n$ is noted as $t_{n}=n \Delta t$ when $n=0,1, \ldots t_{0}=0$ represents the initial time. The digital image at time $n$ is $s_{x, y}^{n}=s\left(x, y, t_{n}\right)$. Thus, we have $s_{x, y}^{0}=s_{0}\left(x, y, t_{0}\right)+n\left(x, y, t_{0}\right)$, where $s_{x, y}^{0}$ is the original image and $s_{0}$ is the desired clean image that is a constant, that is, $s_{0}\left(x, y, t_{0}\right)=s_{0}\left(x, y, t_{n}\right)$. We can derive the numerical implementation of fractional calculus about time from (1) as

$$
\frac{\partial^{v} s}{\partial t^{v}}=\Delta t^{-v}\left[s_{x, y}^{n+1}-s_{x, y}^{n}+\frac{2 \mu \eta}{\Gamma(3-v)}\left(s_{x, y}^{n}-s_{x, y}^{v *}\right)^{2}\left(s_{x, y}^{n}\right)^{-v}\right]
$$

when $v \neq 1$,

where $\mu$ and $\eta$ are the factors for ensuring iterative convergence. Also, the desired clean image $s_{0}\left(x, y, t_{0}\right)$ is unknown, but the intermediate denoising results is an approximate to the desired clean image, that is, $s_{x, y}^{n} \rightarrow s_{0}\left(x, y, t_{0}\right)=$ $s_{0}\left(x, y, t_{n}\right)$. We consider that $\left(s-s_{0}\right)_{x, y} \cong s_{x, y}^{0}-s_{x, y}^{n}$ for making $s$ close to $s_{0}$ as much as possible. We should compute the fractional calculus on the 8 directions simultaneously in practice in order to improve the calculation accuracy and antirotation capability.

The best image $s_{x, y}^{v *}$ in (36) is unknown in numerical iteration, but the intermediate result $s_{x, y}^{n}$ is an approximate to $s_{x, y}^{\nu *}$, that is, $s_{x, y}^{n} \rightarrow s_{x, y}^{\nu *}$. We consider that $\left(s_{x, y}^{n}-\right.$ $\left.s_{x, y}^{v *}\right)^{2} \cong\left(s_{x, y}^{n-1}-s_{x, y}^{n}\right)^{2}$ to make the iterative result approximate $\left(s_{x, y}^{n}-s_{x, y}^{v *}\right)^{2}$ as much as possible. Take $\eta=1$ in (36) and $k=0,1$ in (34) and (35), then we can derive the numerical implementation of (34) and (35) as

$$
\begin{aligned}
s_{x, y}^{n+1}= & P\left(s_{x, y}^{n}\right) \Delta t^{v_{3}}-\frac{\lambda^{n} \Delta t^{v_{3}}}{\Gamma\left(1-v_{3}\right) \Gamma\left(2-v_{3}\right)} s_{x, y}^{n} \\
& +s_{x, y}^{n}-\frac{2 \mu}{\Gamma\left(3-v_{3}\right)}\left(s_{x, y}^{n-1}-s_{x, y}^{n}\right)^{2}\left(s_{x, y}^{n}\right)^{-v_{3}}
\end{aligned}
$$

when $v_{3} \neq 1,2$ and 3 ,

$$
\lambda^{n}=\frac{\Gamma\left(1-v_{3}\right) \Gamma\left(2-v_{3}\right)}{\sigma^{n^{2}} s_{x, y}^{n}} \sum_{x, y} P\left(s_{x, y}^{n}\right)\left(s_{x, y}^{0}-s_{x, y}^{n}\right)^{2},
$$

where $\prod_{\tau=1}^{n}\left(v_{2}-\tau+1\right) \stackrel{n=0}{=} 1, \sigma^{n^{2}}=\sum_{x, y}\left(s_{x, y}^{0}-s_{x, y}^{n}\right)^{2}$, $P\left(s_{x, y}^{n}\right)=\left(-\Gamma\left(1-v_{1}\right) / \Gamma\left(-v_{1}\right) \Gamma\left(-v_{3}\right)\right) \sum_{k=0}^{1}\left(\prod_{\tau=1}^{2 k}\left(v_{2}-\right.\right.$ $\tau+1) /(2 k) !)\left[D_{x}^{1}\left(\left|D^{v_{1}} s_{x, y}^{n}\right|^{v_{2}-2 k-2} D_{x}^{v_{1}} s_{x, y}^{n}\right)+D_{y}^{1}\left(\left|D^{v_{1}} s_{x, y}^{n}\right|^{v_{2}-2 k-2}\right.\right.$ $\left.\left.\cdot D_{y}^{v_{1}} s_{x, y}^{n}\right)\right]$.

We should pay attention to the following when performing numerical iterative implementation. First, $\mu$ is a small number in (37) to ensure convergence, and here we take $\mu=0.005$. Second, we do not need to know or estimate the variance of noise, but we need to assume $\sigma^{1^{2}}$ to be a small positive number in the first iteration. We therefore assume that $\sigma^{1^{2}}=0.01$ in the experiment below. We take $\sigma^{12}$ to (38) and perform numerical iteration. Each iterative result $\sigma^{n^{2}}$ may be different, but it is the approximate to the variance of noise. Third, it is possible that $\left|D^{v_{1}} s_{x, y}^{n}\right|=0$ in numerical iterative calculation, so we take $\left|D^{v_{1}} s_{x, y}^{n}\right|=0.0689$ when $\left|D^{v_{1}} s_{x, y}^{n}\right| \leq 0.0689$ to ensure that (37) and (38) are meaningful. Fourth, we take $s_{x, y}^{n}=0.00001$ when $s_{x, y}^{n}=0$ to make $\left(s_{x, y}^{n}\right)^{-v_{3}}$ have meaning. Fifth, to completely denoise faint noise in very low-frequency and direct current, FDM takes the simple way by reducing the convex in the area where gradient is not changed obviously. We therefore need to perform low-pass filtering for very low-frequency and direct current in numerical iterating. The practices of (37) and (38) are as follows. For one-dimensional signal, we consider that $s_{x}^{n+1}=\left(s_{x-1}^{n+1}+2 s_{x}^{n+1}+s_{x+1}^{n+1}\right) / 4$ when $\left|D^{v_{1}} s_{x}^{n}\right| \prec \alpha_{A}$ and $\alpha_{N A} \prec \alpha_{A}$ and if the noise is not severe in order to ensuring denoising effect, and in the rest conditions we consider that $s_{x}^{n+1}=$ $s_{x}^{n+1}$, where $\alpha_{A}=\left(1 / N_{x}\right) \sum_{x}^{N_{x}}\left|D^{v_{1}} s_{x}^{n}\right|, \alpha_{N A}=\left(\left|D^{v_{1}} s_{x-1}^{n}\right|+\right.$ $\left.2\left|D^{v_{1}} s_{x}^{n}\right|+\left|D^{v_{1}} s_{x+1}^{n}\right|\right) / 4$, and if the noise is very strong, we consider that $s_{x}^{n+1}=\left(s_{x-1}^{n+1}+2 s_{x}^{n+1}+s_{x+1}^{n+1}\right) / 4$ to accelerate the denoising speed. For two-dimensional signal, we consider $s_{x, y}^{n+1}=\left({ }^{x} s_{x, y}^{n+1}+{ }^{y} s_{x, y}^{n+1}+{ }^{r} s_{x, y}^{n+1}+{ }^{l} s_{x, y}^{n+1}\right) / 4$. When $\alpha_{N A}^{x}=$ $\min \left(\alpha_{N A}^{x}, \alpha_{N A}^{y}, \alpha_{N A}^{r}, \alpha_{N A}^{l}\right)$, they are ${ }^{x} s_{x, y}^{n+1}=\left(s_{x-1, y}^{n+1}+2 s_{x, y}^{n+1}+\right.$ $\left.s_{x+1, y}^{n+1}\right) / 4$ and ${ }^{y} s_{x, y}^{n+1}={ }^{r} s_{x, y}^{n+1}={ }^{l} s_{x, y}^{n+1}=s_{x, y}^{n+1}$, when $\alpha_{N A}^{y}=$ $\min \left(\alpha_{N A}^{x}, \alpha_{N A}^{y}, \alpha_{N A}^{r}, \alpha_{N A}^{l}\right)$ they are ${ }^{y} s_{x, y}^{n+1}=\left(s_{x, y-1}^{n+1}+2 s_{x, y}^{n+1}+\right.$ $\left.s_{x, y+1}^{n+1}\right) / 4$ and ${ }^{x} s_{x, y}^{n+1}={ }^{r} s_{x, y}^{n+1}={ }^{l} s_{x, y}^{n+1}=s_{x, y}^{n+1}$, when $\alpha_{N A}^{r}=$ $\min \left(\alpha_{N A}^{x}, \alpha_{N A}^{y}, \alpha_{N A}^{r}, \alpha_{N A}^{l}\right)$ they are ${ }^{r} s_{x, y}^{n+1}=\left(s_{x-1, y+1}^{n+1}+2 s_{x, y}^{n+1}+\right.$ $\left.s_{x+1, y-1}^{n+1}\right) / 4$ and ${ }^{x} s_{x, y}^{n+1}={ }^{y} s_{x, y}^{n+1}={ }^{l} s_{x, y}^{n+1}=s_{x, y}^{n+1}$, and in the rest occasions, it has ${ }^{x} s_{x, y}^{n+1}={ }^{y} s_{x, y}^{n+1}={ }^{r} s_{x, y}^{n+1}={ }^{l} s_{x, y}^{n+1}=$ $s_{x, y}^{n+1}$, where $\alpha_{N A}^{x}=\left(\left|D^{v_{1}} s_{x-1, y}^{n}\right|+2\left|D^{v_{1}} s_{x, y}^{n}\right|+\left|D^{v_{1}} s_{x+1, y}^{n}\right|\right) / 4$, $\alpha_{N A}^{y}=\left(\left|D^{v_{1}} s_{x, y-1}^{n}\right|+2\left|D^{v_{1}} s_{x, y}^{n}\right|+\left|D^{v_{1}} s_{x, y+1}^{n}\right|\right) / 4$, and $\alpha_{N A}^{r}=\left(\left|D^{v_{1}} s_{x-1, y+1}^{n}\right|+2\left|D^{v_{1}} s_{x, y}^{n}\right|+\left|D^{v_{1}} s_{x+1, y-1}^{n}\right|\right) / 4, \alpha_{N A}^{l}=$ $\left(\left|D^{v_{1}} s_{x-1, y-1}^{n}\right|+2\left|D^{v_{1}} s_{x, y}^{n}\right|+\left|D^{v_{1}} s_{x+1, y+1}^{n}\right|\right) / 4$. Here, $x$ denotes $x$-coordinate direction, $y$ denotes $y$-coordinate direction, $r$ denotes right diagonal direction, and $l$ denotes left diagonal direction. Sixth, we expand the edge of the image for better denoising edge pixels. Seventh, since we perform low-pass 


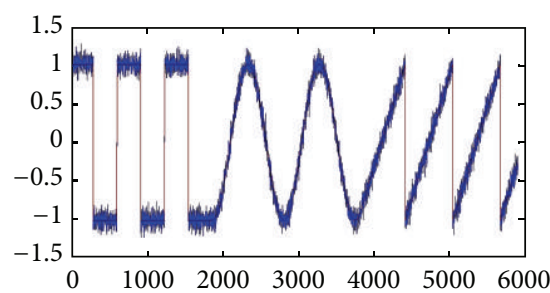

_ Noisy signal

— Original clean signal

(a)

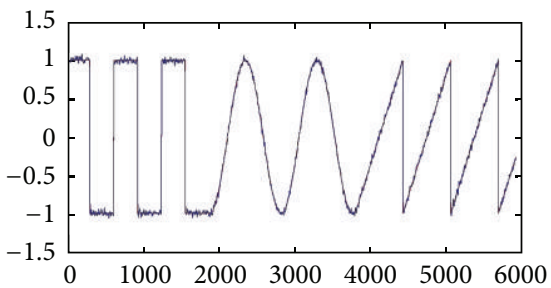

- Original clean signal

— Bilateral filtering denoising

(d)

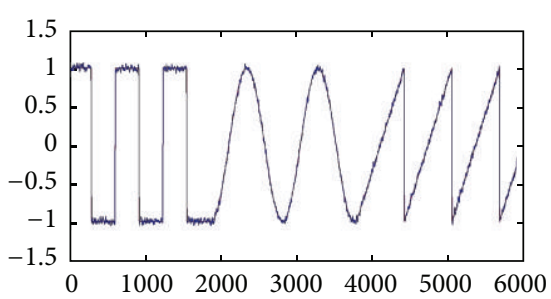

- Original clean signal
NLMF denoising

(g)

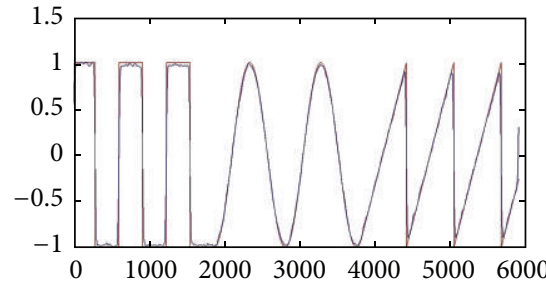

- Original clean signal

— Gaussian denoising

(b)

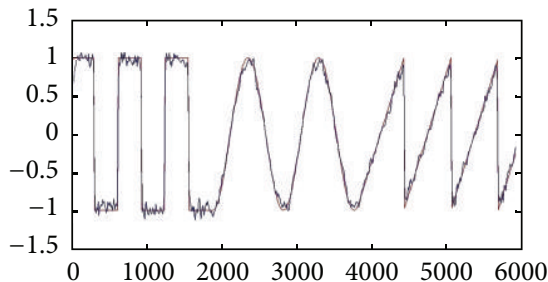

- Original clean signal

— Contourlet denoising

(e)

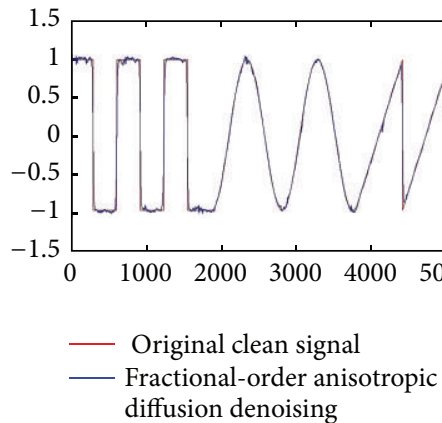

(h)

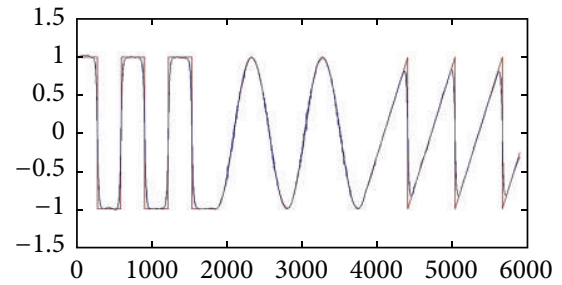

- Original clean signal

- Fourth-order TV denoising

(c)

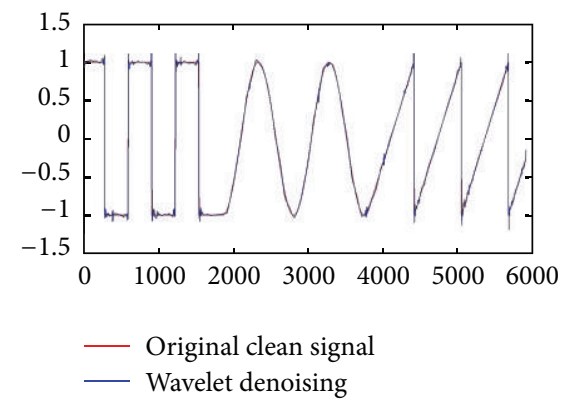

(f)

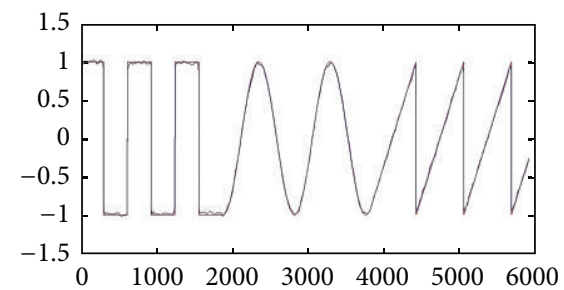

- Original clean signal

(i)

FIGURE 4: Denoising for integrated one-dimensional signal comprising rectangle wave, sine wave, and sawtooth wave. (a) Original clean signal and noisy signal (adds white Gaussian noise to the original clean signal, peak signal-to-noise ratio (PSNR) $=25.2486$ ), (b) Gaussian denoising, (c) fourth-order TV denoising [71-73], (d) bilateral filtering denoising [86-88], (e) contourlet denoising [89, 90], (f) wavelet denoising [88, 91], (g) nonlocal means noise filtering (NLMF) denoising [92, 93], (h) fractional-order anisotropic diffusion denoising [82], and (i) FDM denoising $\left(v_{1}=1.025, v_{2}=2.25, v_{3}=1.05, \Delta t=0.0296\right)$.

filtering in (37) and (38), in order to remove the possible divergence point in numerical iteration, we consider that $s_{x}^{n+1}=s_{x}^{n}$ when $\left|s_{x}^{n+1}\right|>6\left|s_{x}^{n}\right|$ for one-dimensional signal, and we consider that $s_{x, y}^{n+1}=s_{x, y}^{n}$ when $\left|s_{x, y}^{n+1}\right|>6\left|s_{x, y}^{n}\right|$ for twodimensional signal.

4.2. Denoising Capabilities Analysis of the Fractional Partial Differential Equation-Based Denoising Model for Texture Image. To analyze and explain the denoising capabilities of fractional partial differential equation-based denoising model for texture image, we perform the comparative experiments using the composite one-dimensional signal combined by the rectangle wave, the sine wave and the sawtooth wave. The numerical iteration will stop at the point where the peak signal-to-noise ratio (PSNR) is the highest, as shown in Figure 4.

From subjective visual effect we know the following from Figure 4. First, the denoising effect of Gaussian denoising and fourth-order TV denoising is comparatively worse than others, and the high-frequency singularity component has been greatly diffused and smoothed. We can see from Figures 4(b) and 4(c) that the convexes of high-frequency edge of rectangle wave and sawtooth wave are remarkably smoothed and their energy of high-frequency singularity is obviously diffused in neighboring. Second, the denoising capability of fractional-order anisotropic diffusion denoising is in the middle; that is, the capability of maintaining high-frequency singularity is better than that of Gaussian denoising, fourthorder TV denoising, and contourlet denoising but less than 
bilateral filtering denoising, wavelet and NLMF denoising. Also, the denoising is not completed. We can see from Figure 4(h) that the convexes of high-frequency edge of the rectangle wave and the sawtooth wave are weakly smoothed, and their energy is weakly diffused in neighboring. Also, the denoised signal has tiny burr. Third, the denoising capabilities of bilateral filtering denoising, contourlet denoising, wavelet denoising, and NLMF denoising are better, which can well maintain the high-frequency singularity but the denoising is still uncomplete. We can see from Figures $4(\mathrm{~d})-4(\mathrm{~g})$ that the convexes of high-frequency edge of rectangle wave and sawtooth wave are well retained, but the denoised signal still has many small burrs. Finally, the denoising capability of FDM is the best, which is not only well maintains the highfrequency edge of rectangle wave and sawtooth wave but also denoises completely. We can see from Figure 4(i) that the high-frequency edge singularity of rectangle and sawtooth wave is well maintained and little burr is left.

From the viewpoint of quantitative analysis, we take PSNR and correlation coefficients between noisy signal or denoised signal and original clean signal to measure the denoising effect of the above algorithms, as shown in Table 1.

We know from Table 1 as follows. First, PSNR of Gaussian denoising, fourth-order TV denoising, and contourlet denoising are relatively small among the above approaches with $26.9611 \leq$ PSNR $\leq 28.2945$ and their correlation coefficients are also small with $0.9953 \leq$ correlation coefficients $\leq$ 0.9959, which indicates that their denoising capability is worse and the similarity between denoised signal and original clean signal is also low. Second, PSNR of fractional-order anisotropic diffusion denoising is in the middle with PSNR = 29.8692, and its correlation coefficients are in the middle with correlation coefficients $=0.9975$, which shows that its denoising capability and the similarity between denoised signal and original clean signal is also in the middle. Third, PSNR of bilateral filtering denoising, wavelet denoising, NLMF denoising, and FDM denoising is relatively big with $33.3088 \leq$ PSNR $\leq 39.0434$, and its correlation coefficients are also big with $0.9975 \leq$ correlation coefficients $\leq 0.9996$, which shows that their denoising capabilities are better and the similarity between denoised signal and original clean signal is also high. PSNR and correlation coefficients of FDM denoising is the highest, which shows that its denoising capability is the best and the similarity between denoised signal and original clean signal is also the highest.

From the subjective visual of Figure 4 and quanlitative analysis of Table 1, we know the following. First, FDM has the best denoising capability no matter in highfrequency, middle-frequency, and low-frequency components, the denoised signal fits for the edge of high-frequency and the outline of middle-frequency component, and also the noise in low-frequency component is removed clearly and little blur is left. Second, the high-frequency edge singularity of rectangle signal and sawtooth wave signal in Figure 4 has strong high-frequency component which corresponds to high-frequency edge and texture details of two-dimensional signal, while the high-frequency singularity is small and the middle-frequency components are big in the slope of sine wave and sawtooth signal which corresponds to
TABLE 1: Denoising effect for composite one-dimensional signal that combined rectangle wave, sine wave, and sawtooth wave.

\begin{tabular}{lcc}
\hline Denoising algorithm & \multicolumn{2}{c}{$\begin{array}{c}\text { Denoising effect } \\
\text { correlation Coefficients }\end{array}$} \\
\hline Noisy signal & 25.2486 & 0.9951 \\
Gaussian denoising & 26.9611 & 0.9953 \\
Fourth-order TV denoising & 28.2589 & 0.9959 \\
Bilateral filtering denoising & 35.0247 & 0.9990 \\
Contourlet denoising & 28.2945 & 0.9959 \\
Wavelet denoising & 32.1565 & 0.9975 \\
NLMF denoising & 33.3088 & 0.9976 \\
Fractional-order anisotropic & 29.8692 & 0.9975 \\
Diffusion denoising & 39.0434 & 0.9996 \\
FDM denoising & & \\
\hline
\end{tabular}

middle-frequency of two-dimensional signal. The constant direct current of regulation signal corresponds to lowfrequency and direct current components and backgrounds.

To analyze and explain the good denoising capability of FDM, we choose the better models, including bilateral filtering denoising, wavelet denoising, NLMF denoising, and FDM denoising to perform the contrast experiments for texture-rich metallographic images of an iron ball. The numerical iterative process will stop at the point where the PSNR is the highest, as shown in Figure 5.

From a subjective view of the visual effect, we know the following from Figure 5. First, the denoising capabilities of bilateral filtering and wavelet denoising are worse than the other methods, because they obviously diffuse and smooth the high-frequency edge and texture details. We can see that the edge and texture details are clear from Figures 5(e) and $5(\mathrm{~h})$; that is, the denoised noise by bilateral filtering and wavelet denoising may not be the same as the added noise. And we can see from Figures 5(f) and 5(i) that the denoised image is blurry. The denoising is not completed in Figure 5(i), which shows that their capabilities for preserving edge and texture details are worse. Second, the capability for preserving edge and texture details of NLMF denoising is better, but its denoising capability for edge and texture neighboring is worse. From Figure 5(k), we know that though the edge and texture details of denoised image are weaker than those in Figures 5(e) and 5(h), but they still can be seen, the denoised noise by NLMF denoising is close to the added noise. In Figure 5(1), the edge and texture details are small blurred, that is, NLMF denoising can well preserve the edge and texture details. Also, the neighboring of edge and texture details is smooth in Figure 5(k), while the residual noise in the edge and texture neighboring is stronger than other parts in Figure 5(l), that is, the denoising capability of NLMF denoising is worse at the edge and texture neighboring. Third, the denoising capabilities of FDM denoising are the best, which preserve the high-frequency edge and texture details well and also denoise comparatively completed. We can see indistinctly the edge and texture detail from Figures 5(n) and 5(q), which shows that the denoised noise by FDM denoising 


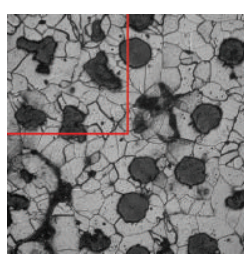

(a)

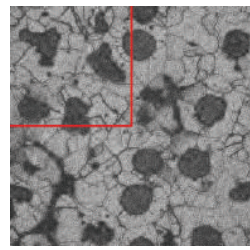

(g)

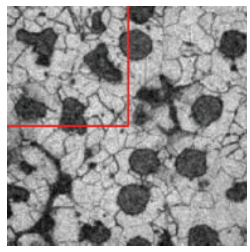

(m)

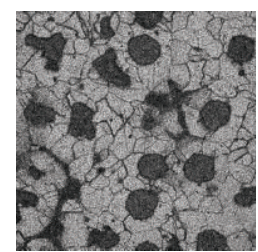

(b)

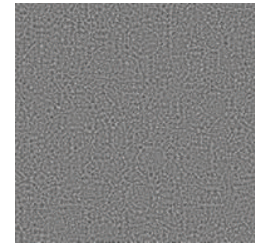

(h)

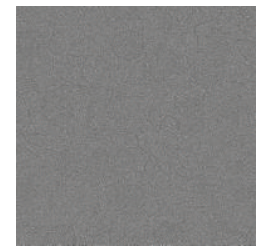

(n)

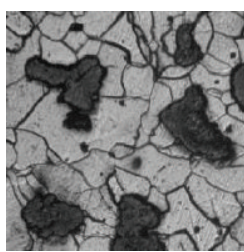

(c)

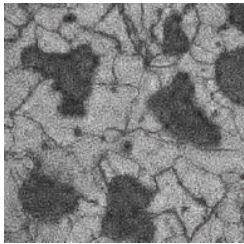

(i)

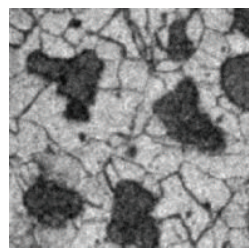

(o)

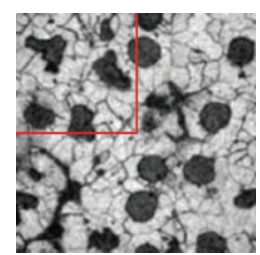

(d)

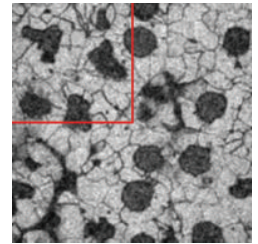

(j)

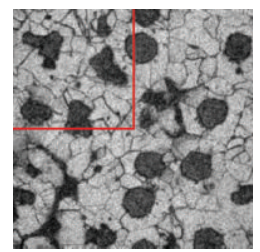

(p)

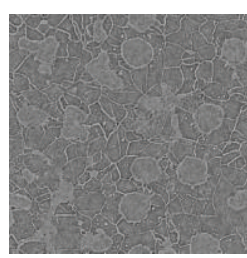

(e)

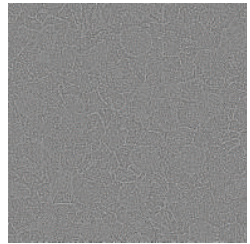

(k)

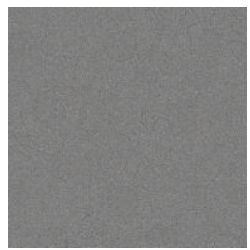

(q)

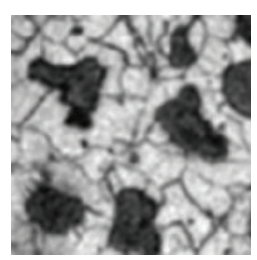

(f)

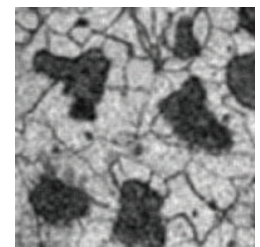

(1)

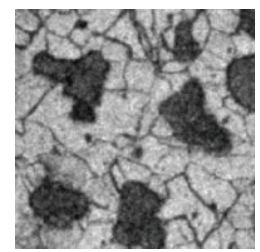

(r)

FIGURE 5: Denoising effects of texture-rich metallographic image of iron ball. (a) Original clean image, (b) noisy image (adds white Gaussian noise to the original clean image, PSNR $=13.6872$ ), (c) partial enlarged details of $1 / 4$ party of (a) red box, (d) denoised image of bilateral filtering denoising $[88,89,93]$, (e) residual plot of bilateral filtering denoising, (f) partial enlarged details of $1 / 4$ party of (d) red box, (g) denoised image of wavelet denoising [92, 93], (h) residual plot of wavelet denoising, (i) partial enlarged details of $1 / 4$ party of (g) red box, (j) denoised image of NLMF denoising [94, 95], (k) residual plot of NLMF denoising, (l) partial enlarged details of 1/4 party of (j) red box, (m) denoised image of FDM denoising $\left(v_{1}=1.75, v_{2}=2.25, v_{3}=1.05, \Delta t=10^{-10}\right)$, (n) residual plot of FDM denoising, (o) partial enlarged details of $1 / 4$ party of $(\mathrm{m})$ red box, $(\mathrm{p})$ denoised Image of FDM denoising $\left(v_{1}=2.25, v_{2}=2.5, v_{3}=1.25, \Delta t=10^{-10}\right)$, (q) residual plot of FDM denoising, ( $r$ ) partial enlarged details of $1 / 4$ party of $(\mathrm{p})$ red box.

TABLE 2: Comprehensive denoising effects results for texture-rich metallographic images of an iron ball.

\begin{tabular}{lcccccc}
\hline \multirow{2}{*}{ Denoising algorithm } & \multicolumn{2}{c}{ Denoising effect } \\
& PSNR & Correlation coefficients & Contrast & Correlation & Energy & Homogeneity \\
\hline Noisy image & 13.6872 & 0.9991 & 5.9118 & 0.2491 & 0.0197 & 0.4580 \\
Bilateral filtering denoising & 20.4845 & 0.9995 & 1.1200 & 0.8038 & 0.0787 & 0.7092 \\
Wavelet denoising & 16.5708 & 0.9994 & 2.1387 & 0.3910 & 0.0473 & 0.5833 \\
NLMF denoising & 21.0494 & 0.9996 & 1.2524 & 0.7150 & 0.0728 & 0.6822 \\
FDM denoising & 21.4826 & 0.9996 & 1.4365 & 0.6876 & 0.0688 & 0.6646 \\
FDM denoising & 21.5482 & 0.9997 & 1.4371 & 0.6646 & 0.0688 & 0.6608 \\
\hline
\end{tabular}

is the most close to the added noise; the edge and texture details are the smallest blurred and the noise is the clearest removed in Figures 5(o) and 5(r), that is, the capabilities for preserving the edge and texture details of FDM are the best.

From the viewpoint of quantitative analysis, we take the PSNR, the correlation coefficients between the noisy image or the denoised image and the original clean image [94], and the average gray level concurrence matrix to comprehensively estimate the denoised effect. We calculate the gray level concurrence matrix coefficient in 5 pixel distance in Figure 5 and export the typical coefficients: contrast, correlation, energy, and homogeneity taking four directions of $0^{\circ}, 45^{\circ}, 90^{\circ}$, and $135^{\circ}$. Here, $0^{\circ}$ represents the projection in the positive $y$-coordinate direction, and $90^{\circ}$ represents the projection in the $x$-coordinate direction. We then average the above values, as seen in Table 2 .

We know the denoising capabilities of the above algorithms are as follows from Table 2. First, the denoising capabilities of bilateral filtering and wavelet denoising are worse than the other methods and their PSNR values and correlation coefficients are relatively small. This shows that the high-frequency edge and texture details are greatly diffused and smoothed and the noise is not completely removed. The similarity between denoised image and original clean image is small. Also, the contrast of average gray level concurrence matrix for bilateral filtering denoising is small, 


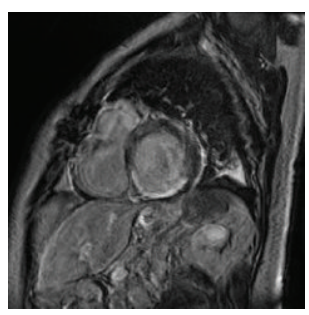

(a)

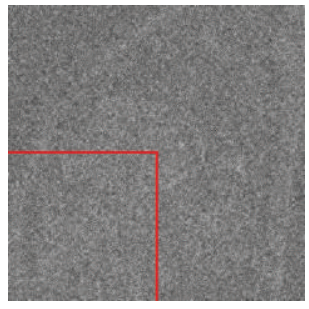

(e)

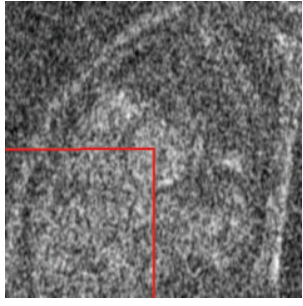

(i)

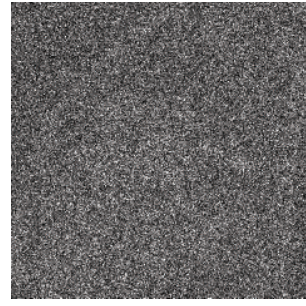

(b)

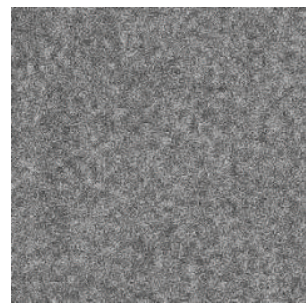

(f)

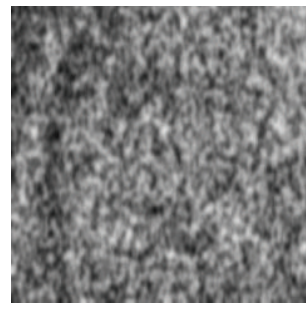

(j)

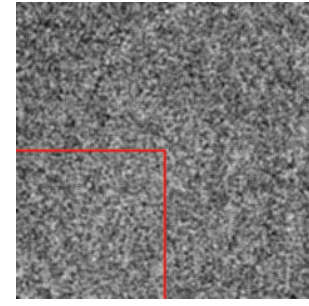

(c)

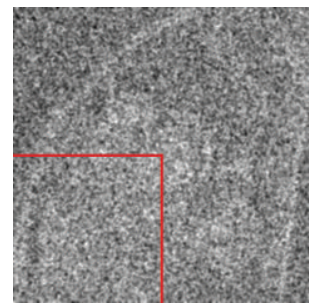

(g)

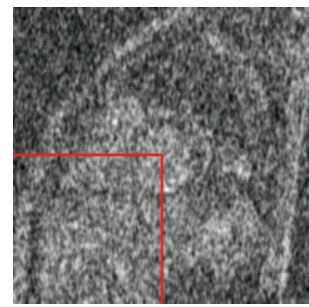

(k)

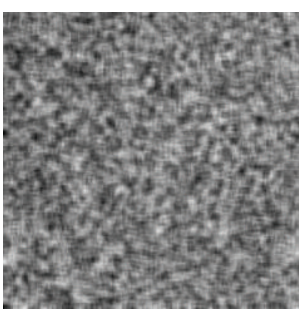

(d)

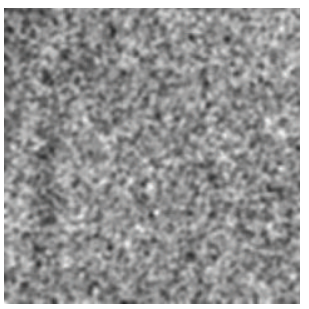

(h)

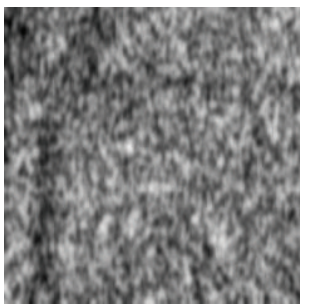

(l)

FIGURE 6: Denoising effects of abdomen MRI of texture-rich internal organ, when Gaussian noise is very strong. (a) Original clean MRI, (b) noisy image (adds white Gaussian noise to the original clean MRI, PSNR $=5.4389$ ), (c) denoised image of bilateral filtering denoising $[88,89,93]$, (d) partial enlarged details of $1 / 4$ party of (c) red box, (e) denoised image of wavelet denoising [88, 91], (f) partial enlarged details of 1/4 party of (e) red box, (g) denoised image of NLMF denoising [94, 95], (h) partial enlarged details, 1/4 party of (g) red box, (i) denoised image of FDM denoising $\left(v_{1}=1.75, v_{2}=2.75, v_{3}=1.05, \Delta t=5 \times 10^{-6}\right)$, (j) partial enlarged details of $1 / 4$ party of (i) red box, $(\mathrm{k})$ denoised image of FDM denoising $\left(v_{1}=2.25, v_{2}=2.5, v_{3}=1.25, \Delta t=5 \times 10^{-6}\right)$, and (l) partial enlarged details of $1 / 4$ party of $(\mathrm{k})$ red box.

which shows that fewer pixels have great contrast and that the texture depth is light and seems fuzzy. The contrast of the average gray level concurrence matrix of wavelet denoising is the largest. This shows that there are more pixels with great contrast, but we cannot say that the texture depth is deeper and the visual effects are clearer because the denoising is incomplete. Second, the denoising capabilities of NLMF and FDM denoising are better. Their PSNR and correlation coefficients are comparatively higher, which indicates that the high-frequency edge and texture details of the denoised image are well preserved, that the denoising is completed, and that the similarity between the denoised image and original clean image is also great. PSNR and correlation coefficients of FDM denoising are the highest, that is, the denoising is most completed and the similarity is also the greatest. The contrast of the average gray level concurrence matrix of FDM denoising is the highest. This shows that there are more pixels with great contrast, that the texture depth is the deepest, and that the image looks clearer. The correlation is small, which shows that the partial gray correlation is weak and that the texture details are obvious. The energy is rather smaller, which shows that the texture changing is not uniform and regular, and thus the texture details are obvious. The homogeneity is also small, which indicates the regional changing is dramatic and that the texture details are obvious. Therefore, we can conclude that FDM denoising is the best denoising algorithm.

To consider a scenario where the Gaussian noise is very strong, and especially when the original clean signal is completely drowned in noises, we perform comparison experiments using the well-performed algorithms discussed above, including bilateral filtering denoising, wavelet denoising, NLMF denoising, and FDM denoising for further analysis of the denoising capability of FMD for robust noise. The numerical iterative process will also stop at the point where peak signal-to-noise ratio is the highest, as seen in Figure 6.

From the viewpoint of visual effects, we know the following from Figure 6, when noise is very strong, especially when MRI is drawn completely. First, the denoising capabilities of bilateral filtering and wavelet denoising are worse than the other methods. We can see indistinctly that the contour and the texture details of inner organ can hardly be recognized from Figures 6(c)-6(f). Second, the denoising capability of NLMF is better because we see that the contour is clearer, but 
TABLE 3: Denoising effects of abdomen MRI of texture-rich internal organ, when Gaussian noise is very strong.

\begin{tabular}{lcccccc}
\hline \multirow{2}{*}{ Denoising algorithm } & \multicolumn{5}{c}{ Denoising Effect } \\
& PSNR & Correlation coefficients & Contrast & Correlation & Energy & Homogeneity \\
\hline Noisy Image & 5.4389 & 0.9857 & 21.2697 & 0.0013 & 0.0844 & 0.4811 \\
Bilateral filtering Denoising & 11.4039 & 0.9894 & 1.1790 & 0.1796 & 0.1113 & 0.6562 \\
Wavelet denoising & 9.9246 & 0.9892 & 1.8443 & 0.0175 & 0.0806 & 0.5941 \\
NLMF denoising & 12.5855 & 0.9921 & 1.1795 & 0.1247 & 0.1083 & 0.6406 \\
FDM denoising & 17.4317 & 0.9975 & 1.1847 & 0.0836 & 0.0913 & 0.5737 \\
FDM denoising & 17.4572 & 0.9976 & 1.1853 & 0.0830 & 0.0893 & 0.5715 \\
\hline
\end{tabular}

the edge and texture details are still blurry as in Figures $6(\mathrm{~g})$ and 6(h). Finally, the denoising capabilities of FDM denoising are the best because we can see from Figures 6(i)-6(l) that the contour is clearest and the edge and texture details can be recognized also.

For quantitative analysis, we measure the denoising effects in terms of the PSNR, the correlation coefficients between the noisy image or the denoised image and the original clean MRI [94], and the average gray level concurrence matrix, as seen in Table 3.

From Table 3, we know that the denoising capabilities of the above algorithms are as follows, when noise is very strong, especially when MRI is completely drowned by the noise. First, the denoising capabilities of bilateral filtering denoising and wavelet denoising are rather poor, and their PSNR and correlation coefficients are relatively small. This shows that the noise cannot be clearly denoised and the similarity between the denoised image and the original clean MRI is small. Also, the contrast of average gray level concurrence matrix of wavelet algorithm is the greatest, which indicates greater pixels with greater contrast but we cannot say that the texture depth is deeper and that the visual effects are clearer because the denoising is incomplete. Second, the denoising capabilities of NLMF denoising and FDM denoising are better and their PSNR and correlation coefficients are comparatively higher, which shows that the noise is denoised completely and the similarity between denoised image and original clean MRI is great. The PSNR and the correlation coefficients of FDM denoising is the highest and its contrast of average gray level concurrence matrix is the greatest. Also, its correlation, energy, and homogeneity are smaller. We therefore can conclude that FDM denoising is the best model of the above models.

When the noise is very strong and especially when original clean signal is completely drowned in noises, we take a texture-rich meteorite crater remote sensing image of moon satellite to perform further comparison experiments using the above well-performed algorithms, including bilateral filtering denoising, wavelet denoising, NLMF denoising, and FDM denosing to test the denoising capability of FDM for robust noise. The added noise is the composite noise combined by white Gaussian noise, salt and pepper noise, and speckle noise. Also, the numerical iterative process will stop at the point where peak signal-to-noise ratio is the highest, as shown in Figure 7.

From view of visual effects, we know from Figure 7 the following; when the composite noise is added by white
Gaussian noise, salt and pepper noise, and speckle together, especially when texture-rich meteorite crater remote sensing image of moon satellite is completely drown in noises. First, the denoising capabilities of bilateral filtering and wavelet and NLMF denoising are comparatively worse. We can see from Figures $7(\mathrm{c})-7(\mathrm{~h})$ that the contour may be seen indistinctly and the edge and texture details can hardly be recognized. Second, the denoising capability of FDM denoising is the best. We can see from Figures 7(i)-7(l) that the contour is not only the clearest and also the edge and texture details can be clearly recognized.

For quantitative analysis, we measure the denoising effects in terms of the PSNR, correlation coefficients between noisy image or denoised image and original clean remote sensing image [94], and average gray level concurrence matrix, as seen in Table 4 .

We know denoising capabilities of the above algorithms from Table 4 are as follows, when composite noise added by white Gaussian noise, salt and pepper noise, and speckle noise, especially when the original image is completely drowned in noises. First, the denoising capabilities of bilateral filtering, wavelet, and NLMF denoising are poor, and their PSNR and correlation coefficients are relatively small. This shows that the added noise cannot be denoised completely and the similarities between denoised image and original clean MRI are small. Also, the contrasts of average gray level concurrence matrices of NLMF algorithm are the greatest, which indicates that there are more pixels with great contrast, but we cannot say that the texture depth is deeper and the visual effect is clearer because the denoising is uncompleted. Second, the denoising capability of FDM denoising is the best. Its PSNR and correlation coefficients are comparatively high, which shows that the noise is completely denoised and the similarity between denoised image and original clean MRI is the greatest. Also, the PSNR and the correlation coefficients of FDM denoising are the highest. The contrast of average gray level concurrence matrix of FDM denoising is high, while its correlation, energy, and homogeneity are small. We therefore can say that FDM denoising is the best denoising model of the above models.

By comparing the visual effects of Figures 5, 6, and 7 and quantitative analysis in Figures 1, 2, and 3, we find the following. First, the denoising capability of FDM is the best, irrespective of the strength and type of added noise. Its PSNR and correlation coefficients are the highest and the denoising is relatively completed. The similarity between the denoised image and the original image is the highest. Second, 


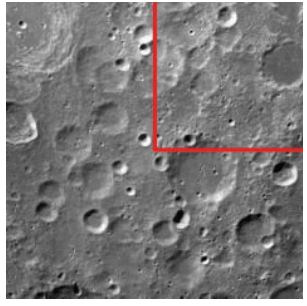

(a)

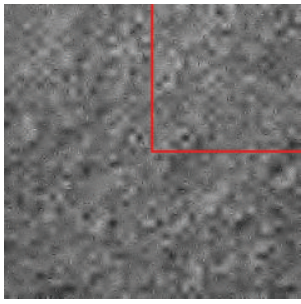

(e)

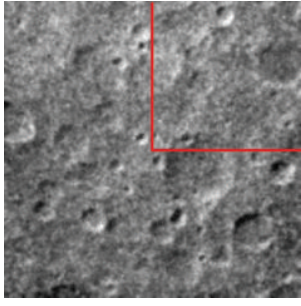

(i)

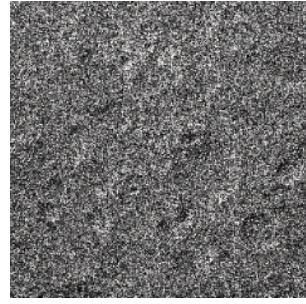

(b)

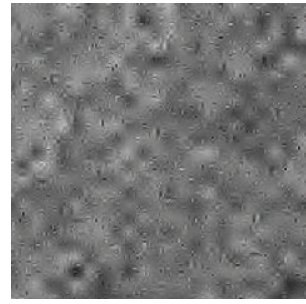

(f)

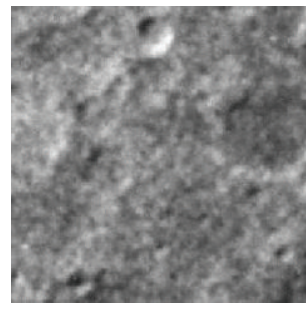

(j)

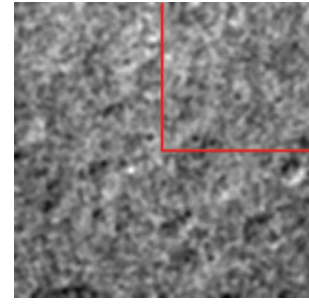

(c)

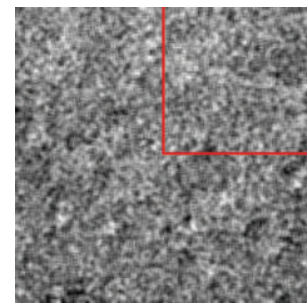

(g)

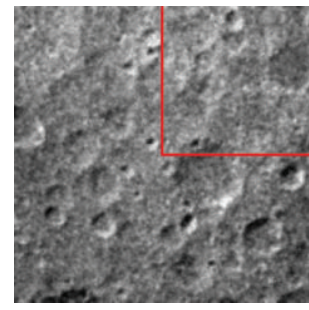

(k)

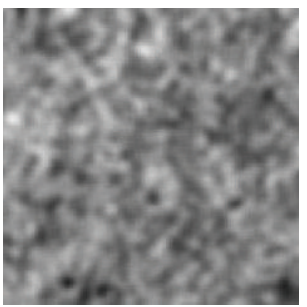

(d)

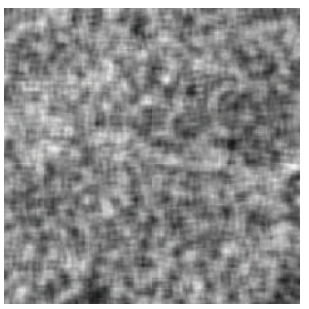

(h)

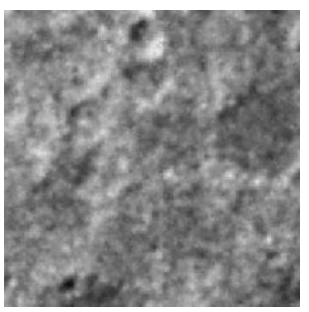

(l)

FIGURE 7: Denoising effects of meteorite crater texture-rich moon satellite remote sensing image, when white Gaussian noise, salt, and pepper noise and speckle noise are added together. (a) Original clean image, (b) noisy image (adds white Gaussian noise (its standard variance is 0.02), salt and pepper noise (its noise density is 0.2 ), and speckle noise (its standard variance is 0.1 ) to the original clean image, PSNR $=8.8564$ ), (c) denoised image of bilateral filtering denoising [88, 89, 93], (d) partial enlarged details of 1/4 party of (c) red box, (e) denoised image of wavelet denoising [92, 93], (f) partial enlarged details of $1 / 4$ party of (e) red box, (g) denoised image of NLMF denoising [94, 95], (h) partial enlarged details of $1 / 4$ party of $(\mathrm{g})$ red box, (i) denoised image of FDM denoising $\left(v_{1}=1.75, v_{2}=2.75, v_{3}=1.05\right.$, and $\left.\Delta t=10^{-10}\right)$, (j) partial enlarged details of $1 / 4$ party of (i) red box, (k) denoised image of FDM denoising $\left(v_{1}=2.25, v_{2}=2.5, v_{3}=1.25\right.$, and $\left.\Delta t=10^{-10}\right)$, and $(\mathrm{l})$ Partial enlarged details of $1 / 4$ party of $(\mathrm{k})$ red box.

TABLE 4: Denoising effects on texture-rich meteorite crater remote sensing image of moon satellite, when white Gaussian noise, salt and pepper noise and speckle noise are added together.

\begin{tabular}{lcccccc}
\hline \multirow{2}{*}{ Denoising algorithm } & \multicolumn{2}{c}{ Denoising effect } \\
& PSNR & Correlation coefficients & Contrast & Correlation & Energy & Homogeneity \\
\hline Noisy image & 8.8564 & 0.9968 & 12.5054 & 0.0159 & 0.0230 & 0.3930 \\
Bilateral filtering denoising & 19.3530 & 0.9991 & 0.4934 & 0.6371 & 0.1601 & 0.7841 \\
Wavelet denoising & 18.3278 & 0.9990 & 0.5736 & 0.7302 & 0.2348 & 0.7765 \\
NLMF denoising & 19.9585 & 0.9989 & 1.0104 & 0.4319 & 0.0999 & 0.6797 \\
FDM denoising & 21.1448 & 0.9995 & 0.61033 & 0.5881 & 0.1476 & 0.7598 \\
FDM denoising & 21.1455 & 0.9996 & 0.6609 & 0.5809 & 0.1363 & 0.7495 \\
\hline
\end{tabular}

FDM denoising can nonlinearly preserve the low-frequency contour feature in the smooth area to the furthest degree, nonlinearly enhance high-frequency edge information in those areas where gray level changes obviously, nonlinearly enhance the high-frequency edge and texture details, and also nonlinearly maintain the fractional multiscale denoising capability in those areas where gray level does not change obviously.

\section{Conclusions}

We propose the introduction of a new mathematical method-fractional calculus to the field of image processing and the implementation of fractional partial differential equation. First, it presents three common-used definitions of Grümwald-Letnikov, Riemann-Liouville, and Caputo, which is the premise of fractional partial differential equation-based 
denoising model. Second, we derive fractional Green's formula for two-dimensional image processing by extending classical integer order to fractional order and then derive fractional Euler-Lagrange formula. Based on the above fractional formulas, a fractional partial differential equation is proposed. Finally, we show the denoising capability of the proposed model by comparing Gaussian denoising, fourthorder TV denoising, bilateral filtering denoising, contourlet denoising, wavelet Denoising, nonlocal means noise filtering (NLMF) denoising, and fractional-order anisotropic diffusion denoising. The experimental results prove that FDM can preserve the low-frequency contour feature in the smooth area, nonlinearly maintain the high-frequency edge and texture details in those areas where gray level change greatly and also nonlinearly retain the texture details in those areas where gray level has little changed. As for texture-rich images, the denoising capability of the proposed FDM denoising model is obviously superior to traditional integral based algorithm when denoising.

\section{Acknowledgment}

The work is supported by Foundation Franco-Chinoise Pour La Science Et Ses Applications (FFCSA), China National Nature Science Foundation (Grants no. 60972131 and no. 61201438), Returned Overseas Chinese Scholars, State Education Ministry of China (Grant no. 20111139), Sichuan Science and Technology Support Project of China (Grant nos. 2011GZ0201 and 2013SZ0071), Open fund of State key Laboratory of Networking and Switching Technology of Beijing University of Posts and Telecommunications (SKLNST-20101-03), and Project of Sichuan Province of China under Grant 2011GZ0201and Grant 2013SZ0071, the Soft Science Project of Sichuan Province of China under Grant 2013ZR0010, and the initial funding for young teachers of Sichuan University under Grant 2011SCU11.

\section{References}

[1] E. R. Love, "Fractional derivatives of imaginary order," Journal of the London Mathematical Society, vol. 3, pp. 241-259, 1971.

[2] K. B. Oldham and Spanier, The Fractional Calculus: Integrations and Differentiations of Arbitrary Order, Academic Press, New York, NY, USA, 1974.

[3] A. C. McBride, Fractional Calculus, Halsted Press, New York, NY, USA, 1986.

[4] K. Nishimoto, Fractional Calculus, University of New Haven Press, New Haven, Conn, USA, 1989.

[5] S. G. Samko, A. A. Kilbas, and O. I. Marichev, Fractional Integrals and Derivatives: Theory and Applications, Gordon and Breach Science Publishers, Yverdon, Switzerland, 1993.

[6] K. S. Miller, "Derivatives of noninteger order," Mathematics Magazine, vol. 68, no. 3, pp. 183-192, 1995.

[7] S. G. Samko, A. A. Kilbas, and O. I. Marichev, Fractional Integrals and Derivatives: Theory and Applications, Gordon and Breach Science, Yverdon, Switzerland, 1987.

[8] N. Engheta, "On fractional calculus and fractional multipoles in electromagnetism," Institute of Electrical and Electronics
Engineers. Transactions on Antennas and Propagation, vol. 44, no. 4, pp. 554-566, 1996.

[9] N. Engheta, "On the role of fractional calculus in electromagnetic theory," IEEE Antennas and Propagation Magazine, vol. 39, no. 4, pp. 35-46, 1997.

[10] M.-P. Chen and H. M. Srivastava, "Fractional calculus operators and their applications involving power functions and summation of series," Applied Mathematics and Computation, vol. 81, no. 2-3, pp. 287-304, 1997.

[11] P. L. Butzer and U. Westphal, "An introduction to fractional calculus," in Applications of Fractional Calculus in Physics, chapter 1, pp. 1-85, World Scientific, Singapore, 2000.

[12] S. Kempfle, I. Schäfer, and H. Beyer, "Fractional calculus via functional calculus: theory and applications," Nonlinear Dynamics, vol. 29, no. 1-4, pp. 99-127, 2002.

[13] R. L. Magin, "Fractional calculus in bioengineering," Critical Reviews in Biomedical Engineering, vol. 32, no. 3-4, pp. 195-377, 2004.

[14] A. A. Kilbas, H. M. Srivastava, and J. J. Trujiilo, Theory and Applications of Fractional Differential Equations, Elsevier, Amsterdam, The Netherlands, 2006.

[15] J. Sabatier, O. P. Agrawal, and J. A. Tenreiro Machado, Advances in Fractional Calculus: Theoretical Developments and Applications in Physics and Engineering, Springer, Dordrecht, The Netherlands, 2007.

[16] R. C. Koeller, "Applications of fractional calculus to the theory of viscoelasticity," Journal of Applied Mechanics, vol. 51, no. 2, pp. 299-307, 1984.

[17] Y. A. Rossikhin and M. V. Shitikova, "Applications of fractional calculus to dynamic problems of linear and nonlinear hereditary mechanics of solids," Applied Mechanics Reviews, vol. 50, no. 1, pp. 15-67, 1997.

[18] S. Manabe, "A suggestion of fractional-order controller for flexible spacecraft attitude control," Nonlinear Dynamics, vol. 29, no. 1-4, pp. 251-268, 2002.

[19] W. Chen and S. Holm, "Fractional Laplacian time-space models for linear and nonlinear lossy media exhibiting arbitrary frequency power-law dependency," Journal of the Acoustical Society of America, vol. 115, no. 4, pp. 1424-1430, 2004.

[20] E. Perrin, R. Harba, C. Berzin-Joseph, I. Iribarren, and A. Bonami, "Nth-order fractional Brownian motion and fractional Gaussian noises," IEEE Transactions on Signal Processing, vol. 49, no. 5, pp. 1049-1059, 2001.

[21] C.-C. Tseng, "Design of fractional order digital FIR differentiators," IEEE Signal Processing Letters, vol. 8, no. 3, pp. 77-79, 2001.

[22] Y. Q. Chen and B. M. Vinagre, "A new IIR-type digital fractional order differentiator," Signal Processing, vol. 83, no. 11, pp. 2359$2365,2003$.

[23] Y.-F. Pu, Research on application of fractional calculus to latest signal analysis and processing [Ph.D. thesis], Sichuan University, Chengdu, China, 2006.

[24] Y.-F. Pu, X. Yuan, K. Liao, Z.-L. Chen, and J.-L. Zhou, "Five numerical algorithms of fractional calculus applied in modern signal analyzing and processing," Journal of Sichuan University, vol. 37, no. 5, pp. 118-124, 2005.

[25] Y.-F. Pu, X. Yuan, K. Liao et al., "Structuring analog fractance circuit for $1 / 2$ order fractional calculus," in Proceedings of the IEEE 6th International Conference on ASIC (ASICON '05), vol. 2, pp. 1136-1139, Shanghai, China, October 2005.

[26] Y.-F. Pu, X. Yuan, K. Liao, and J.-L. Zhou, "Implement any fractional order neural-type pulse oscillator with net-grid type 
analog fractance circuit," Journal of Sichuan University, vol. 38, no. 1, pp. 128-132, 2006.

[27] R. Duits, M. Felsberg, L. Florack et al., "Scale spaces on a bounded domain," in Proceedings of the 4th International Conference Scale Spaces, pp. 494-510, Isle of Skye, UK, 2003.

[28] S. Didas, B. Burgeth, A. Imiya, and J. Weickert, "Regularity and scale-space properties of fractional high order linear filtering," in Proceedings of the 5th International Conference on Scale Space and PDE Methods in Computer Vision, Scale-Space, vol. 3459, pp. 13-25, April 2005.

[29] M. Unser and T. Blu, "Fractional splines and wavelets," SIAM Review, vol. 42, no. 1, pp. 43-67, 2000.

[30] B. Ninness, "Estimation of 1/f noise," IEEE Transactions on Information Theory, vol. 44, no. 1, pp. 32-46, 1998.

[31] B. Mathieu, P. Melchior, A. Oustaloup, and C. Ceyral, "Fractional differentiation for edge detection," Signal Processing, vol. 83, no. 11, pp. 2421-2432, 2003.

[32] S.-C. Liu and S. Chang, "Dimension estimation of discrete-time fractional Brownian motion with applications to image texture classification," IEEE Transactions on Image Processing, vol. 6, no. 8, pp. 1176-1184, 1997.

[33] Y.-F. Pu, "Fractional calculus approach to texture of digital image," in Proceedings of the IEEE 8th International Conference on Signal Processing (ICSP '06), pp. 1002-1002, Beijing, China, November 2006.

[34] Y.-F. Pu, "Fractional differential filter of digital image," Invention Patent of China, No.ZL200610021702.3, 2006.

[35] Y.-F. Pu, "High precision fractional calculus filter of digital image," Invention Patent of China, No.ZL201010138742.2, 2010.

[36] Y.-F. Pu, W. Wang, and J.-L. Zhou, "Fractional differential approach to detecting textural features of digital image and its fractional differential filter implementation," Science in China Series F, vol. 38, no. 12, pp. 2252-2272, 2008.

[37] Y.-F. Pu and J.-L. Zhou, "A novel approach for multi-scale texture segmentation based on fractional differential," International Journal of Computer Mathematics, vol. 88, no. 1, pp. 58-78, 2011.

[38] Y.-F. Pu, J.-L. Zhou, and X. Yuan, "Fractional differential mask: a fractional differential-based approach for multiscale texture enhancement," IEEE Transactions on Image Processing, vol. 19, no. 2, pp. 491-511, 2010.

[39] T. Chan, S. Esedoglu, F. Park et al., "Recent developments in total variation image restoration," in Mathematical Models of Computer Vision, Springer, New York, NY, USA, 2005.

[40] A. Buades, B. Coll, and J. M. Morel, "A review of image denoising algorithms, with a new one," Multiscale Modeling \& Simulation, vol. 4, no. 2, pp. 490-530, 2005.

[41] J. Weickert, Anisotropic Diffusion in Image Processing, European Consortium for Mathematics in Industry, B. G. Teubner, Stuttgart, Germany, 1998.

[42] G. Aubert and P. Kornprobst, Mathematical Problems in Image Processing: Partial Differential Equationsand the Calculus of Variations, vol. 147 of Applied Mathematical Sciences, Springer, New York, NY, USA, 2nd edition, 2006.

[43] P. Perona and J. Malik, "Scale-space and edge detection using anisotropic diffusion," IEEE Transactions on Pattern Analysis and Machine Intelligence, vol. 12, no. 7, pp. 629-639, 1990.

[44] L. I. Rudin, S. Osher, and E. Fatemi, "Nonlinear total variation based noise removal algorithms," Physica D, vol. 60, no. 1-4, pp. 259-268, 1992.
[45] G. Sapiro and D. L. Ringach, "Anisotropic diffusion of multivalued images with applications to color filtering," IEEE Transactions on Image Processing, vol. 5, no. 11, pp. 1582-1586, 1996.

[46] P. Blomgren and T. F. Chan, "Color TV: total variation methods for restoration of vector-valued images," IEEE Transactions on Image Processing, vol. 7, no. 3, pp. 304-309, 1998.

[47] N. P. Galatsanos and A. K. Katsaggelos, "Methods for choosing the regularization parameter and estimating the noise variance in image restoration and their relation," IEEE Transactions on Image Processing, vol. 1, no. 3, pp. 322-336, 1992.

[48] S. Z. Li, "Close-form solution and parameter selection for convex minimization-based edge-preserving smoothing," IEEE Transactions on Pattern Analysis and Machine Intelligence, vol. 20, no. 9, pp. 916-932, 1998.

[49] N. Nguyen, P. Milanfar, and G. Golub, "Efficient generalized cross-validation with applications to parametric image restoration and resolution enhancement," IEEE Transactions on Image Processing, vol. 10, no. 9, pp. 1299-1308, 2001.

[50] D. M. Strong, J. F. Aujol, and T. F. Chan, "Scale recognition, regularization parameter selection, and Meyer's $\mathrm{G}$ norm in total variation regularization," Multiscale Modeling \& Simulation, vol. 5, no. 1, pp. 273-303, 2006.

[51] A. M. Thompson, J. C. Brown, J. W. Kay, and D. M. Titterington, "A study of methods of choosing the smoothing parameter in image restoration by regularization," IEEE Transactions on Pattern Analysis and Machine Intelligence, vol. 13, no. 4, pp. 326339, 1991.

[52] P. Mrázek and M. Navara, "Selection of optimal stopping time for nonlinear diffusion filtering," International Journal of Computer Vision, vol. 52, no. 2-3, pp. 189-203, 2003.

[53] G. Gilboa, N. Sochen, and Y. Y. Zeevi, "Estimation of optimal PDE-based denoising in the SNR sense," IEEE Transactions on Image Processing, vol. 15, no. 8, pp. 2269-2280, 2006.

[54] C. R. Vogel and M. E. Oman, "Iterative methods for total variation denoising," SIAM Journal on Scientific Computing, vol. 17, no. 1, pp. 227-238, 1996.

[55] J. Darbon and M. Sigelle, "Exact optimization of discrete constrained total variation minimization problems," in Proceedings of the 10th nternational Workshop on Combinatorial Image AnalysisI (WCIA '04), pp. 548-557, Auckland, New Zealand, 2004.

[56] J. Darbon and M. Sigelle, "Image restoration with discrete constrained total variation. I. Fast and exact optimization," Journal of Mathematical Imaging and Vision, vol. 26, no. 3, pp. 261-276, 2006.

[57] J. Darbon and M. Sigelle, "Image restoration with discrete constrained total variation. II. Levelable functions, convex priors and non-convex cases," Journal of Mathematical Imaging and Vision, vol. 26, no. 3, pp. 277-291, 2006.

[58] B. Wohlberg and P. Rodriguez, "An iteratively reweighted norm algorithm for minimization of total variation functionals," IEEE Signal Processing Letters, vol. 14, no. 12, pp. 948-951, 2007.

[59] F. Catté, P. L. Lions, J. M. Morel, and T. Coll, "Image selective smoothing and edge detection by nonlinear diffusion," SIAM Journal on Numerical Analysis, vol. 29, no. 1, pp. 182-193, 1992.

[60] Y. Meyer, Oscillating Patterns in Image Processing and in Some Nonlinear Evolution Equations, The American Mathematical Society, Providence, RI, USA, 2001.

[61] D. Strong and T. Chan, "Edge-preserving and scale-dependent properties of total variation regularization," Inverse Problems, vol. 19, no. 6, pp. S165-S187, 2003. 
[62] S. Alliney, "A property of the minimum vectors of a regularizing functional defined by means of the absolute norm," IEEE Transactions on Signal Processing, vol. 45, no. 4, pp. 913-917, 1997.

[63] M. Nikolova, "A variational approach to remove outliers and impulse noise," Journal of Mathematical Imaging and Vision, vol. 20, no. 1-2, pp. 99-120, 2004.

[64] T. F. Chan and S. Esedoḡlu, "Aspects of total variation regularized $L^{1}$ function approximation," SIAM Journal on Applied Mathematics, vol. 65, no. 5, pp. 1817-1837, 2005.

[65] M. Nikolova, "Minimizers of cost-functions involving nonsmooth data-fidelity terms," SIAM Journal on Numerical Analysis, vol. 40, no. 3, pp. 965-994, 2002.

[66] S. Osher, M. Burger, D. Goldfarb, J. Xu, and W. Yin, "An iterative regularization method for total variation-based image restoration," Multiscale Modeling \& Simulation, vol. 4, no. 2, pp. 460-489, 2005.

[67] G. Gilboa, Y. Y. Zeevi, and N. Sochen, "Texture preserving variational denoising using an adaptive fidelity term," in Proceedings of the 2nd IEEE Workshop on Variational, Geometric and Level Set Methods in Computer Vision (VLSM '03), pp. 137-144, Nice, France.

[68] S. Esedoglu and S. J. Osher, "Decomposition of images by the anisotropic Rudin-Osher-Fatemi model," Communications on Pure and Applied Mathematics, vol. 57, no. 12, pp. 1609-1626, 2004.

[69] P. Blomgren, T. F. Chan, and P. Mulet, "Extensions to total variation denoising," in Proceedings of the SPIE on Advanced Signal Processing: Algorithms, Architectures and Implementations VII, vol. 3162, pp. 367-375, San Diego, Calif, USA, July 1997.

[70] P. Blomgren, P. Mulet, T. F. Chan, and C. K. Wong, "Total variation image restoration: numerical methods and extensions," in Proceedings of the International Conference on Image Processing (ICIP '97), pp. 384-387, October 1997.

[71] T. Chan, A. Marquina, and P. Mulet, "High-order total variation-based image restoration," SIAM Journal on Scientific Computing, vol. 22, no. 2, pp. 503-516, 2000.

[72] Y. L. You and M. Kaveh, "Fourth-order partial differential equations for noise removal," IEEE Transactions on Image Processing, vol. 9, no. 10, pp. 1723-1730, 2000.

[73] M. Lysaker, A. Lundervold, and X. C. Tai, "Noise removal using fourth-order partial differential equation with applications to medical magnetic resonance images in space and time," IEEE Transactions on Image Processing, vol. 12, no. 12, pp. 1579-1589, 2003.

[74] G. Gilboa, N. Sochen, and Y. Y. Zeevi, "Image enhancement and denoising by complex diffusion processes," IEEE Transactions on Pattern Analysis and Machine Intelligence, vol. 26, no. 8, pp. 1020-1036, 2004.

[75] A. Chambolle and P.-L. Lions, "Image recovery via total variation minimization and related problems," Numerische Mathematik, vol. 76, no. 2, pp. 167-188, 1997.

[76] S. Osher, A. Solé, and L. Vese, "Image decomposition and restoration using total variation minimization and the $H^{-1}$ norm," Multiscale Modeling \& Simulation, vol. 1, no. 3, pp. 349370, 2003.

[77] M. Lysaker and X. C. Tai, "Iterative image restoration combining total variation minimization and a second-order functional," International Journal of Computer Vision, vol. 66, no. 1, pp. 5$18,2006$.

[78] F. Li, C. Shen, J. Fan, and C. Shen, "Image restoration combining a total variational filter and a fourth-order filter," Journal of
Visual Communication and Image Representation, vol. 18, no. 4, pp. 322-330, 2007.

[79] M. Lysaker, S. Osher, and X.-C. Tai, "Noise removal using smoothed normals and surface fitting," IEEE Transactions on Image Processing, vol. 13, no. 10, pp. 1345-1357, 2004.

[80] F. Dong, Z. Liu, D. Kong, and K. Liu, "An improved LOT model for image restoration," Journal of Mathematical Imaging and Vision, vol. 34, no. 1, pp. 89-97, 2009.

[81] P. Guidotti and J. V. Lambers, "Two new nonlinear nonlocal diffusions for noise reduction," Journal of Mathematical Imaging and Vision, vol. 33, no. 1, pp. 25-37, 2009.

[82] J. Bai and X.-C. Feng, "Fractional-order anisotropic diffusion for image denoising," IEEE Transactions on Image Processing, vol. 16, no. 10, pp. 2492-2502, 2007.

[83] G. Leitmann, The Calculus of Variations and Optimal Control: An Introduction, Springer, New York, NY, USA, 1981.

[84] V. E. Tarasov, "Fractional vector calculus and fractional Maxwell's equations," Annals of Physics, vol. 323, no. 11, pp. 2756-2778, 2008.

[85] W. Rudin, Functional Analysis, 2nd Edition, McGraw-Hill, New York, NY, USA, 1991.

[86] C. Tomasi and R. Manduchi, "Bilateral filtering for gray and color images," in Proceedings of the 1998 IEEE 6th International Conference on Computer Vision, pp. 839-846, January 1998.

[87] M. Zhang and B. K. Gunturk, "Multiresolution bilateral filtering for image denoising," IEEE Transactions on Image Processing, vol. 17, no. 12, pp. 2324-2333, 2008.

[88] H. Yu, L. Zhao, and H. Wang, "Image denoising using trivariate shrinkage filter in the wavelet domain and joint bilateral filter in the spatial domain," IEEE Transactions on Image Processing, vol. 18, no. 10, pp. 2364-2369, 2009.

[89] M. N. Do and M. Vetterli, "The contourlet transform: an efficient directional multiresolution image representation," IEEE Transactions on Image Processing, vol. 14, no. 12, pp. 2091-2106, 2005.

[90] D. D.-Y. Po and M. N. Do, "Directional multiscale modeling of images using the contourlet transform," IEEE Transactions on Image Processing, vol. 15, no. 6, pp. 1610-1620, 2006.

[91] G. Y. Chen and T. D. Bui, "Multiwavelets denoising using neighboring coefficients," IEEE Signal Processing Letters, vol. 10, no. 7, pp. 211-214, 2003.

[92] A. Buades, B. Coll, and J.-M. Morel, "A non-local algorithm for image denoising," in Proceedings of the IEEE Computer Society Conference on Computer Vision and Pattern Recognition (CVPR '05), vol. 2, pp. 60-65, San Diego, Calif, USA, June 2005.

[93] A. Buades, B. Coll, and J.-M. Morel, "Nonlocal image and movie denoising," International Journal of Computer Vision, vol. 76, no. 2, pp. 123-139, 2008.

[94] Z. Wang, A. C. Bovik, H. R. Sheikh, and E. P. Simoncelli, "Image quality assessment: from error visibility to structural similarity," IEEE Transactions on Image Processing, vol. 13, no. 4, pp. 600612, 2004.

[95] M. Abramowitz and I. Stegun, Handbook of Mathematical Functions With Formulas, Graphs and Mathematical Tables, U.S. Department of Commerce, Washington, DC, USA, 1964.

[96] P. R. Halmos, Measure Theory, D. van Nostrand Company, New York, NY, USA, 1950.

[97] M. E. Munroe, Introduction to Measure and Integration, Addison Wesley, London, UK, 1953. 


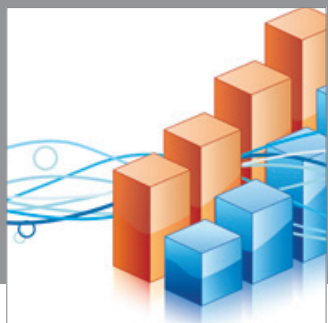

Advances in

Operations Research

mansans

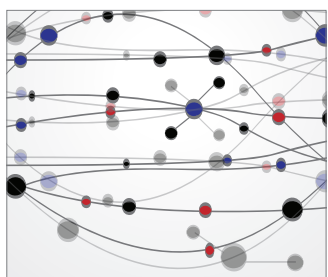

The Scientific World Journal
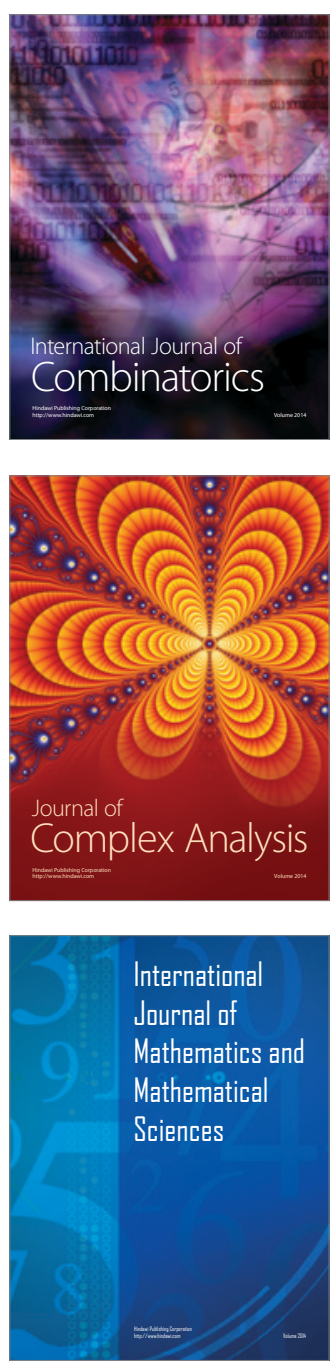
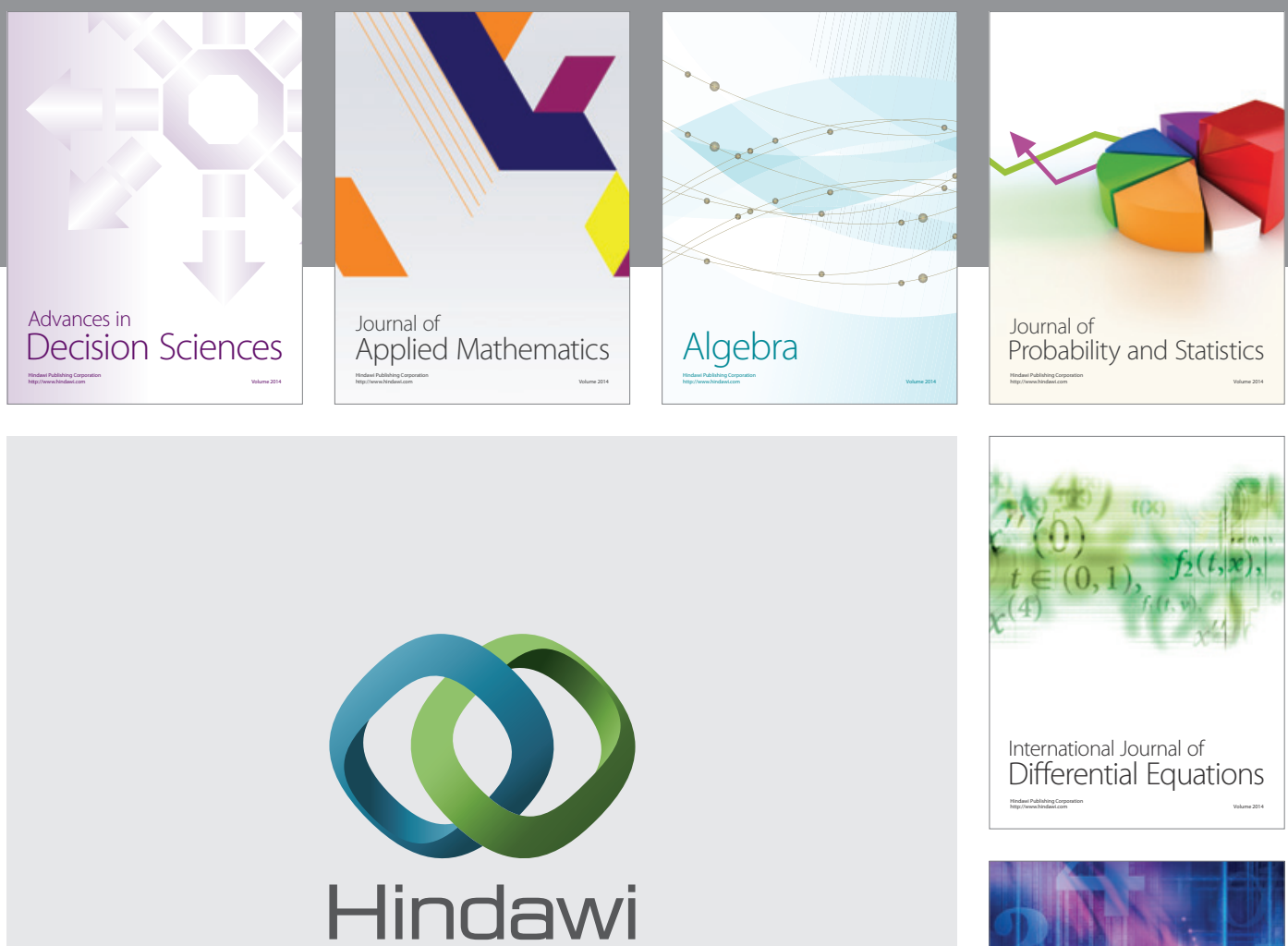

Submit your manuscripts at http://www.hindawi.com
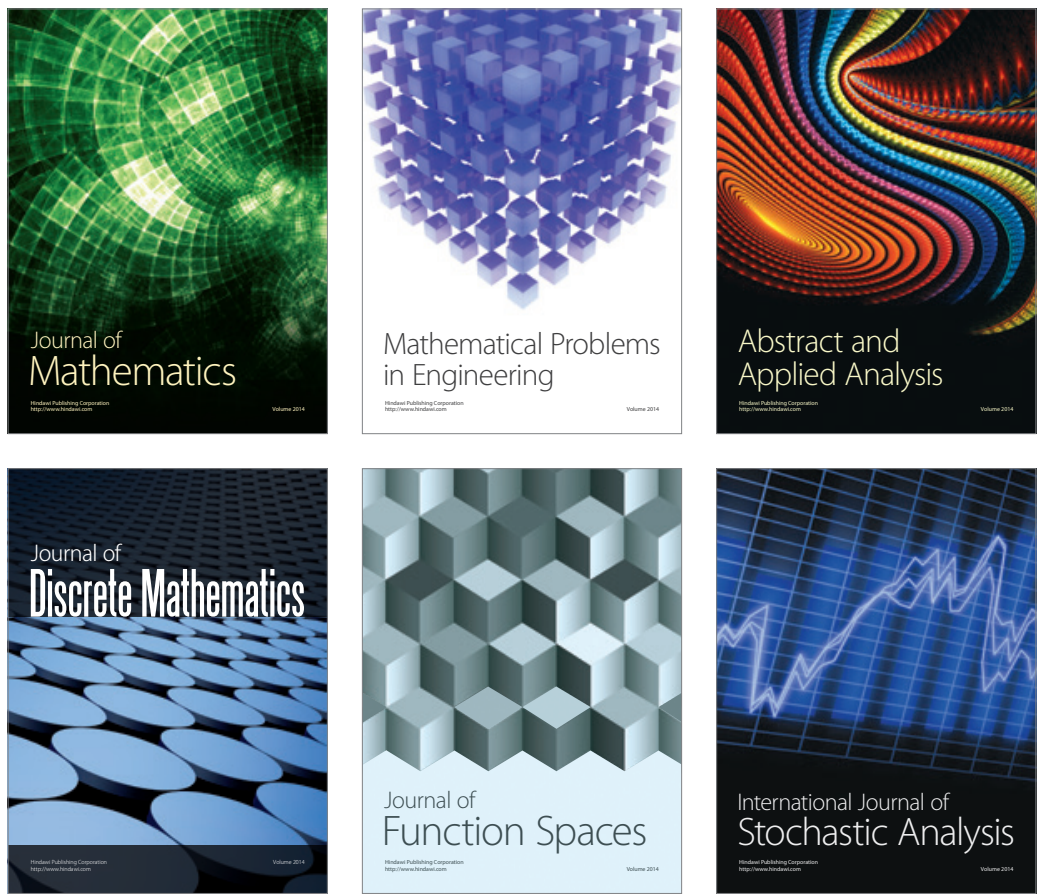

Journal of

Function Spaces

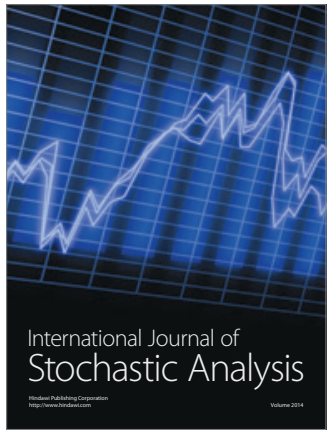

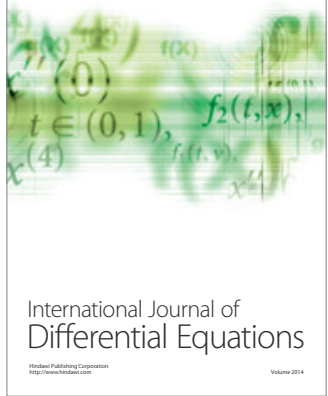
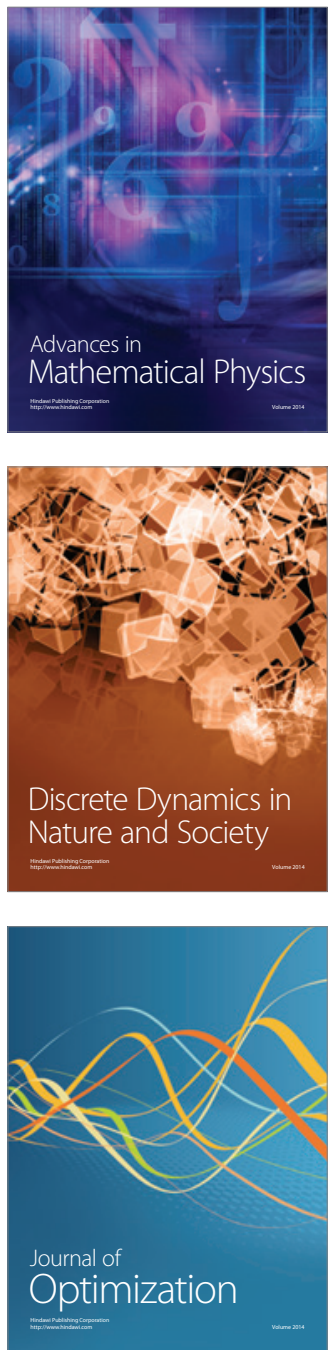